\title{
Reprocessed height time series for GPS stations
}

\author{
S. Rudenko ${ }^{1}$, N. Schön ${ }^{1,2}$, M. Uhlemann ${ }^{1}$, and G. Gendt ${ }^{1}$ \\ ${ }^{1}$ Helmholtz Centre Potsdam GFZ German Research Centre for Geosciences, Telegrafenberg, 14473 Potsdam, Germany \\ ${ }^{2}$ Bristol Glaciology Centre, School of Geographical Sciences, University of Bristol, University Road, Bristol BS8 1SS, UK \\ Correspondence to: S. Rudenko (rudenko@gfz-potsdam.de) and N. Schön (nana.schoen@bristol.ac.uk )
}

Received: 21 June 2012 - Published in Solid Earth Discuss.: 26 July 2012

Revised: 30 November 2012 - Accepted: 10 December 2012 - Published: 24 January 2013

\begin{abstract}
Precise weekly positions of 403 Global Positioning System (GPS) stations located worldwide are obtained by reprocessing GPS data of these stations for the time span from 4 January 1998 until 29 December 2007. The processing algorithms and models used as well as the solution and results obtained are presented. Vertical velocities of 266 GPS stations having a tracking history longer than $2.5 \mathrm{yr}$ are computed; 107 of them are GPS stations located at tide gauges (TIGA observing stations). The vertical velocities calculated in this study are compared with the estimates from the co-located tide gauges and other GPS solutions. The formal errors of the estimated vertical velocities are 0.01$0.80 \mathrm{~mm} \mathrm{yr}^{-1}$. The vertical velocities of our solution agree within $1 \mathrm{~mm} \mathrm{yr}^{-1}$ with those of the recent solutions (ULR5 and ULR3) of the Universite de La Rochelle for about 6775 per cent of the common stations. Examples of typical behaviour of station height changes are given and interpreted. The derived height time series and vertical motions of continuous GPS at tide gauges stations can be used for correcting the vertical land motion in tide gauge records of sea level changes.
\end{abstract}

\section{Introduction}

Satellite radar altimetry and tide gauge measurements are the primary techniques for sea level change investigations. Satellite radar altimetry measures absolute sea level using data obtained during the last $35 \mathrm{yr}$ from altimetry satellite missions GEOS3, SEASAT, GEOSAT, ERS-1, ERS-2, GFO, TOPEX/Poseidon, Jason-1, Envisat, Jason-2, CryoSat-2 and recently HY-2A. Over 1750 tide gauge stations located worldwide measure relative sea level providing long time se- ries (totaling longer than $120 \mathrm{yr}$ ). Therefore, analysis of tide gauge measurements with the purpose of long-term sea level change research requires a well-defined reference frame. Such reference frame can be realized through precise positions and velocities of Global Positioning System (GPS) stations located at or near tide gauges. One of the purposes of the GPS Tide Gauge Benchmark Monitoring (TIGA) Working Group, former Pilot Project (http://adsc.gfz-potsdam.de/ tiga/index_TIGA.html) (Schöne et al., 2009) of the International Global Navigation Satellite System (GNSS) Service (IGS, (Dow et al., 2009)), is to create such a reference frame. The accuracies required by the oceanographic community for sea level studies are about $5-10 \mathrm{~mm}$ for station positions and less than $1 \mathrm{~mm} \mathrm{yr}^{-1}$ for vertical motions (Schöne et al., 2009). Solutions of positions of GPS stations located near to tide gauges were derived by different research groups in 2002-2006 (e.g. Zhang et al., 2008) using relative models for antenna phase centre variations (PCV) and obsolete processing models. However, the switch within the IGS from using a relative to an absolute PCV model mainly affecting the station height, use of new processing software, models and strategies, inclusion of new TIGA GPS stations in the solutions required and made possible a reprocessing within the TIGA project. Within this reprocessing three TIGA analysis centres (a consortium of University of Canberra, University of Tasmania and Australian National University (CTA); German Research Centre for Geosciences (GFZ) and Université de La Rochelle (ULR)) computed global station network solutions. The GFZ and ULR TIGA analysis centres also contributed to the first IGS data reprocessing campaign (IGS repro1, http://acc.igs.org/reprocess.html) and ITRF2008 (Altamimi et al., 2011). 
Various authors used GPS measurements to investigate crustal motions at tide gauges and sea level changes regionally (e.g. Buble et al., 2010; Sanchez and Bosch, 2009) and globally (e.g. Wöppelmann et al., 2007, 2009). Recently, several studies have been made to investigate the influence of various types of systematic errors on station position and vertical velocity estimates. Thus, Munekane and Boehm (2010) investigated troposphere-induced errors in GPS-derived geodetic time series. King and Watson (2010) showed the influence of multipath and geometry effects on long GPS coordinate time series. Effects of azimuthal multipath asymmetry on long GPS coordinate time series were studied by Goebell and King (2011). King et al. (2012) examined the simulated effect of the electromagnetic coupling of a GPS antenna-monument on GPS coordinate time series longer than $2.5 \mathrm{yr}$. Fu et al. (2012) have found $0.3 \mathrm{~mm}$ coordinate differences between solutions using ocean tidal loading (OTL) computed using two different centres of mass: the centre of mass of the Earth system and the centre of mass of the solid Earth. The influence of non-tidal ocean loading effects on geodetic GPS heights has been studied by Williams and Penna (2011). The quality assessment of the recently reprocessed GPS realization of the terrestrial reference frame (Collilieux et al., 2011) shows that the GPS-derived origin is at the centimeter level consistent with the Satellite Laser Ranging (SLR) one with a drift lower than $1 \mathrm{~mm} \mathrm{yr}^{-1}$. From the recently reprocessed ULR solution, Bouin and Wöppelmann (2010) found agreement within $2 \mathrm{~mm} \mathrm{yr}^{-1}$ of the tide gauge measurements and vertical velocities for $84 \%$ of the GPS stations co-located with the tide gauges by analyzing $10 \mathrm{yr}$ of continuous GPS data of more than 200 permanent GPS stations distributed worldwide. Global sea-level rise estimates calculated from tide gauge records corrected using GPS data depend on the terrestrial reference frame used. Thus, errors in the reference frame scale rate and origin rate influence the estimated global sea level rise (Collilieux and Wöppelmann, 2011). That is why generation of a stable reference frame containing precise positions of GPS stations located near to tide gauges is very important.

In this paper, we describe the procedure, models used and the results obtained from the analysis of continuous GPS data from a global network of 403 GPS stations for about a $10-\mathrm{yr}$ time span (1998-2007). Vertical velocities of 266 GPS stations with time series longer than $2.5 \mathrm{yr}$ are computed. We compare our solution with the estimates from co-located tide gauges as well as with the GPS-derived vertical velocities from GFZ previous and external solutions.

The remainder of the paper is organized in the following way. The GPS data processing algorithm and the input data, reference frames and models used are described in Sect. 2. The main results of GPS data reprocessing are presented in Sect. 3. The methodology of station vertical velocity computation and some typical and interesting examples of station height changes are given in Sect. 4. Finally, the results ob-

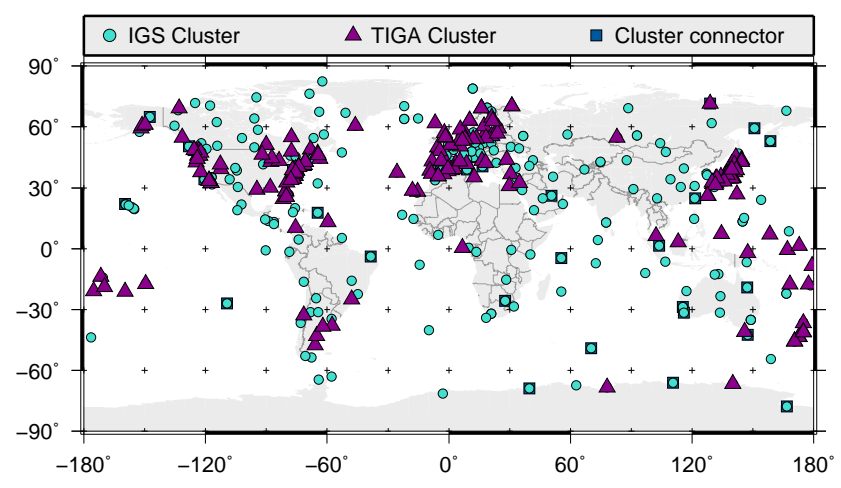

Fig. 1. Global distribution of TIGA and IGS tracking stations used for reprocessing.

tained are discussed (Sect. 5), conclusions drawn and outlook provided.

\section{GPS data processing algorithm}

GPS data of a global network of 403 stations covering time span 4 January 1998 to 29 December 2007 (GPS weeks 939 to 1459) were analysed using EPOS-Potsdam software (Gendt et al. (1994), version 7) recently elaborated. The global network of GPS stations (Fig. 1) used for reprocessing was split in two subnetworks. The first subnetwork includes 216 IGS stations; the second subnetwork includes 187 continuous GPS at tide gauges (TIGA) stations: 107 TIGA observing stations and some other new stations. The subnetworks are combined to form daily solutions using up to 30 distributed worldwide IGS reference stations (cluster connectors) using the procedure described in Zhang et al. (2007) and taking into account the global distribution of the reference stations. All available reference stations were used that were available over the time span. The IGS station subnetwork was used to estimate GPS satellite orbits and clocks that were introduced as fixed values when processing GPS data of the TIGA station subnetwork. To stabilize the GPS satellite orbits, daily solutions are combined into three-day solutions by applying orbit continuity constraints (Beutler et al., 1996). Three-day solutions are combined into weekly solutions using the algorithm outlined in Zhang et al. (2007).

The observation data, reference frames, measurement and orbit models used are described in Table 1. The terrestrial reference frame was defined in the following way. The IGS05 (being an IGS realization of ITRF2005 (Altamimi et al., 2007) for GPS stations) was used as a priori terrestrial reference frame. The initial coordinates of stations present in the IGS05 were taken from the IGS05 and estimated for remaining stations. Coordinates of the reference stations were estimated with tight constraints to their initial values, and loose constraints were used for all other stations. The initial values of station velocities were used from the IGS05, if available, 
Table 1. Input data, reference frames and models used for GPS data processing.

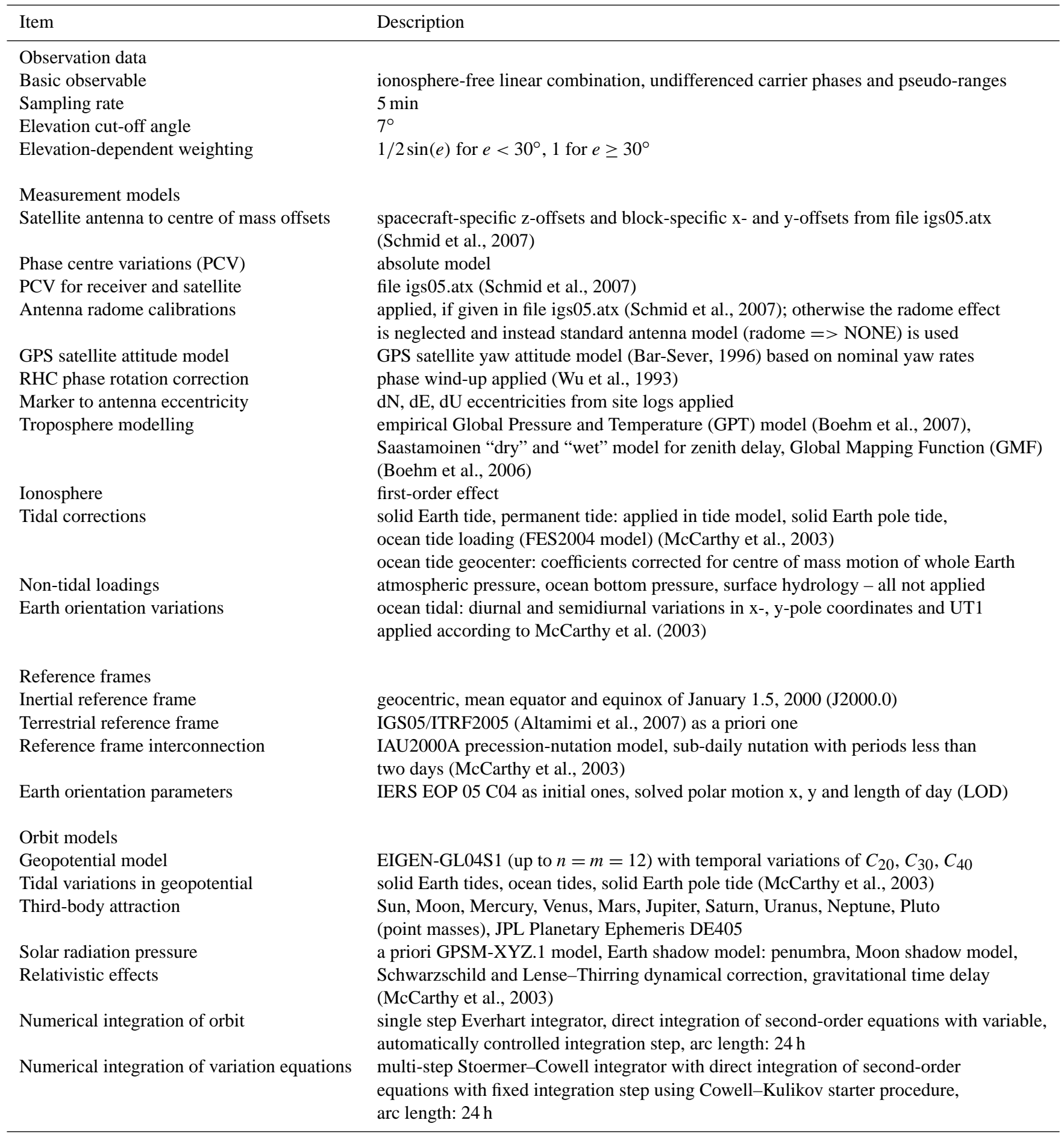

and computed using the NNR-NUVEL1A model (McCarthy et al., 2003) for the remaining stations.

GPS data from up to 300 stations per week were processed using the data processing strategy for huge GNSS global networks (Ge et al., 2006). The following parameters are esti- mated in the least-square adjustment. The Cartesian station coordinates are estimated weekly using free network strategy with constraints $1-1000 \mathrm{~m}$ to a priori values; no station is fixed. Receiver and satellite clocks are solved for at each epoch assuming white noise process. One receiver 


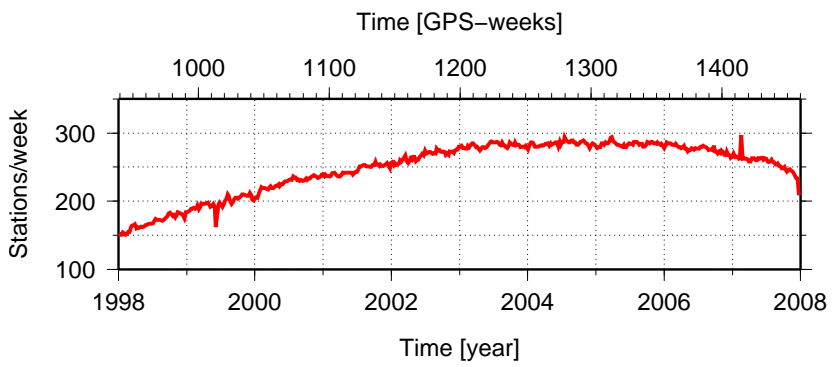

Fig. 2. Number of GPS stations included in the weekly GT1 solutions.

clock is fixed and used as a time reference. Satellite initial position and velocity, solar radiation pressure scale, $y$-bias, sine/cosine terms and stochastic impulses (at noon) are estimated for all satellites once per arc. Yaw rate is adjusted for BLOCK II/IIA satellites during shadow crossing. Troposphere zenith delay coefficients are solved for each station at $1 \mathrm{~h}$ intervals. Troposphere gradients in elevation and azimuth are estimated every $12 \mathrm{~h}$ for each station. Ambiguities are fixed according to Ge et al. (2005). The Earth rotation parameters (ERPs), namely, $x$ and y Earth pole coordinates and their rates and length of day (LOD), are adjusted daily. In a resulting weekly solution, Universal Time UT1 is fixed for the first day of the week and the daily estimates for UT1 and LOD are constrained to obtain a continuous Earth rotation throughout the week.

\section{Results of GPS data reprocessing}

The GFZ TIGA GT1 solution contains weekly coordinates of GPS stations, daily values of $\mathrm{x}$ and $\mathrm{y}$ Earth pole coordinates and their rates and LOD. The solution is available in the Solution (Software/technique) Independent Exchange (SINEX) format via anonymous FTP at TIGA archive (ftp://ftp.gfz-potsdam.de/pub/transfer/kg_igs/igstiga/ solutions/) as files /WWWWW/gftWWWW7.snx.Z and at the Crustal Dynamics Data Information System (CDDIS) (ftp://cddis.gsfc.nasa.gov/gps/products/WWWW/repro1/) as files gt1WWWW7.snx.Z, where WWWW stands for GPS week in the range from 0939 till 1459.

We have up to 300 stations included in the weekly solutions (Fig. 2) for the time interval we considered in this study. This figure also illustrates the rapidly growing observation network in the first years.

As an indicator of relative solution stability, the coordinate repeatabilities of daily solutions with respect to the weekly solution were calculated. The averaged values per week over all stations are depicted in Fig. 3. It is clearly visible that the north and east coordinate components improve over the entire time span and reach even $1 \mathrm{~mm}$ level. The up component looks relatively stable from 2001 onwards and reaches

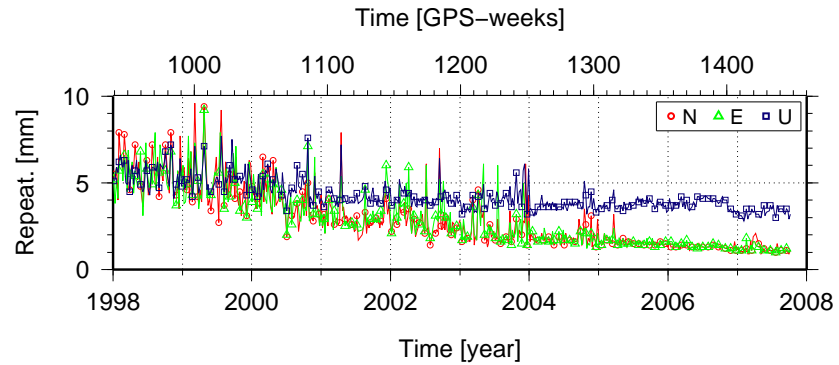

Fig. 3. Weekly overall mean of station coordinate repeatabilities for north $(\mathrm{N})$, east $(\mathrm{E})$ and up $(\mathrm{U})$ components.

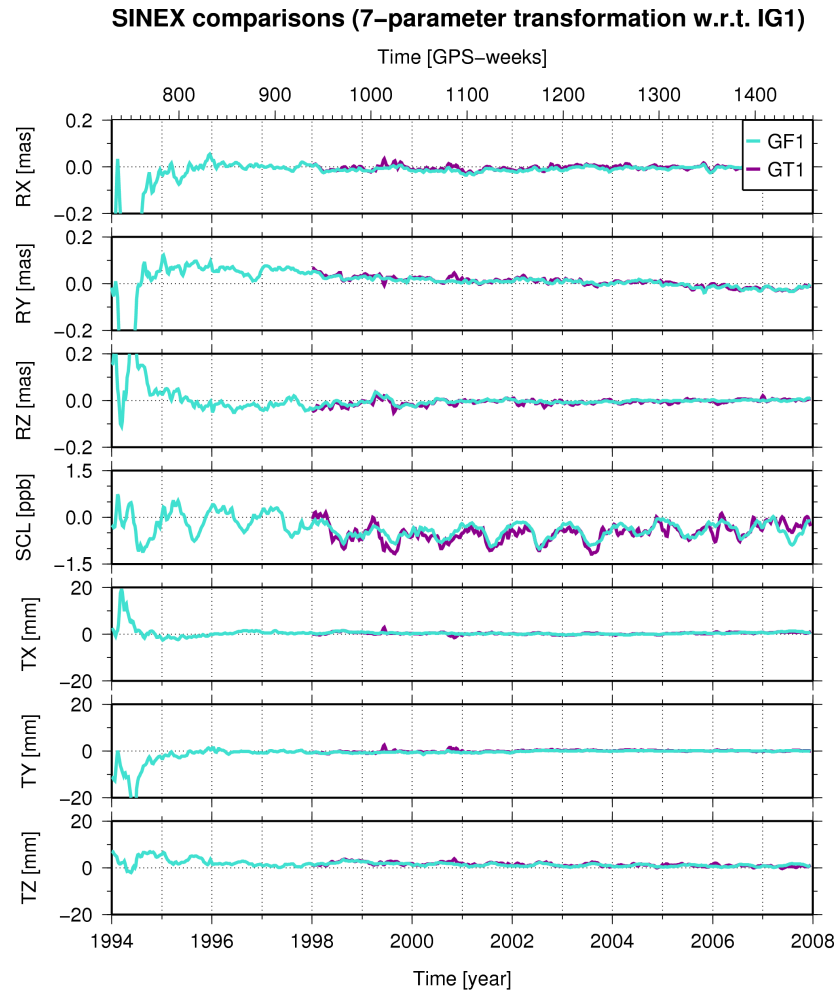

Fig. 4. The time series of weekly Helmert transformation parameters (RX-, RY-, RZ-rotations, TX-, TY-, TZ-translations and scale SCL) of the GT1 and GF1 solutions with respect to the IG1 solution.

$3 \mathrm{~mm}$ level in 2007. These effects can be also related to the enhancements in the station equipment used.

To assess our weekly coordinate solutions, 7-parameter transformations of GT1 solution and GFZ IGS GF1 solution containing only IGS stations were done with respect to the combined IG1 solution of the IGS repro1 campaign available at ftp://cddis.gsfc.nasa.gov/gps/products/repro1/. The time series of weekly Helmert transformation parameters (X-, Y-, Z-rotations, X-, Y-, Z-translations and scale) of the GT1 solution with respect to the IG1 solution are shown in Fig. 4. The average values and standard deviations of the transformation parameters are $-0.004 \pm 0.041,0.007 \pm 0.042$ and $-0.007 \pm$ 


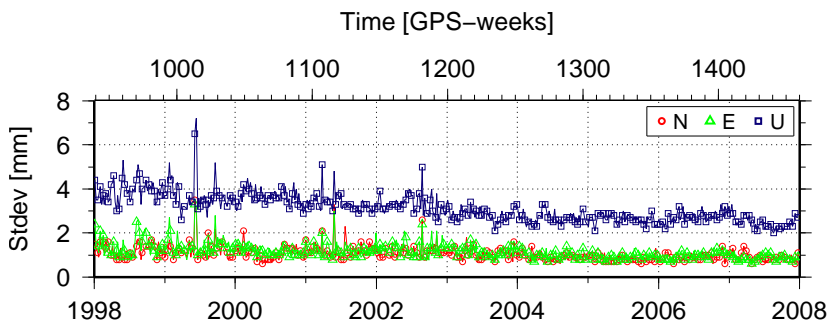

Fig. 5. Standard deviations of the north $(\mathrm{N})$, east $(\mathrm{E})$ and up $(\mathrm{U})$ residual weighted average of the GT1 solution with respect to those of the IG1 combined solution of the IGS repro1 campaign.

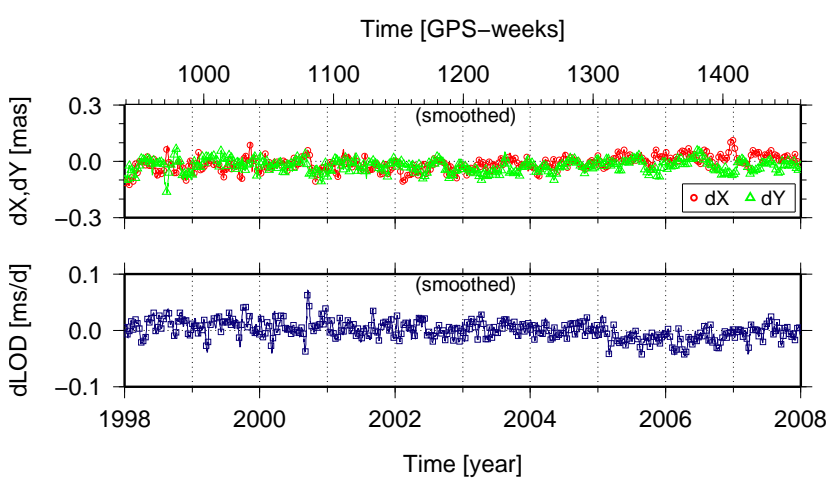

Fig. 6. Comparison of the Earth rotation parameters (X, Y pole coordinates and LOD) of the GT1 solution with respect to those of the IG1 combined solution of the IGS repro1 campaign. The plotted values are smoothed with a sliding 3-day mean.

0.045 mas for X-, Y-, Z-rotations, $0.298 \pm 1.074,-0.045 \pm$ 1.069 and $1.403 \pm 1.056 \mathrm{~mm}$ for $\mathrm{X}-, \mathrm{Y}-, \mathrm{Z}$-translations and $-0.501 \pm 0.166 \mathrm{ppb}$ for scale indicating a good agreement of the GT1 solution with the IG1 solution for the common stations. The standard deviations of the GT1 solution residual weighted average with respect to those of the IG1 solution are plotted in Fig. 5. The accuracy of weekly north and east components is about $1 \mathrm{~mm}$ for the investigated time span, and the accuracy of the up component reaches $3 \mathrm{~mm}$ level at the end of the time interval. Based on these results it can be stated that the required position accuracy needed for the determination of accurate station height time series is fulfilled and that a precise reference frame was obtained.

The daily adjusted values of $\mathrm{x}$ and $\mathrm{y}$ Earth pole coordinates and length of day of the GT1 solution are compared to those of the IG1 combined solution. The good agreement between the two solutions in $\mathrm{x}$ and $\mathrm{y}$ pole coordinates with mean values computed using original daily values and standard deviation (scatter in time series of the ERP differences) of $-0.012 \pm 0.056$ and $-0.026 \pm 0.049$ mas and LOD with $0.000 \pm 0.023 \mathrm{~ms} \mathrm{~d}^{-1}$ can be seen in Fig. 6 .

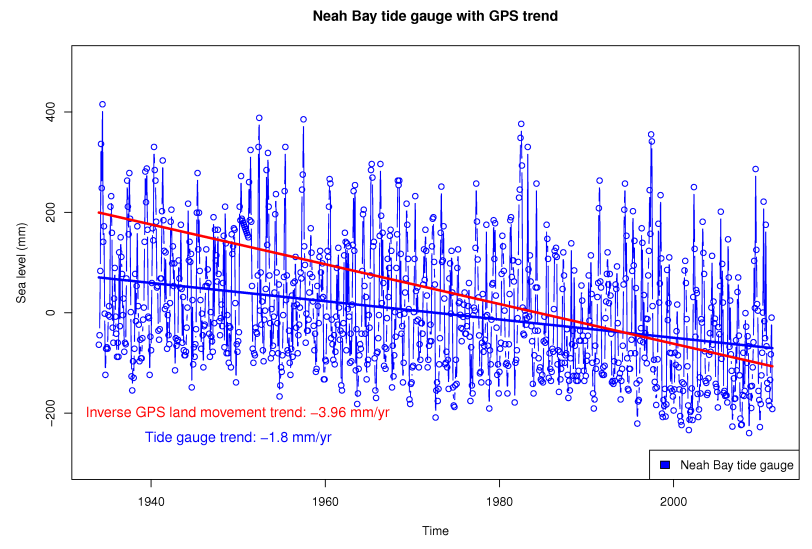

Fig. 7. Height time series and trend for Neah Bay tide gauge and inverse height trend of the co-located GPS station NEAH.

\section{Station height changes}

\subsection{Station vertical velocity computation methodology}

Vertical trends were determined from the GT1 SINEX files by extracting Cartesian station coordinates and converting them to longitude, latitude and height using the WGS84 geoid model. The time series were then fitted to extract a linear trend using the standard deviation values as reciprocal weights to account for measurement errors. Trend changes were determined using the Breaks For Additive Seasonal and Trend (BFAST) package (Verbesselt et al., 2010). The BFAST algorithm uses a four-step iterative procedure to detect breakpoints in time series. First, the ordinary least squares (OLS) residuals-based moving sum (MOSUM) test is used to detect whether breakpoints do occur in the time series. If the test indicates a significant change (at the significance level $p<0.05$ ), the breakpoints are estimated from the seasonally adjusted data. In the second step, the trend is estimated using robust regression. The OLS-MOSUM test is then applied again in the third step to test for breakpoints in the seasonal component of the time series. In the final step, the seasonal component is estimated from the detrended data. The above steps are iterated until the number and position of breakpoints are unchanged. Details regarding the procedure are described in Verbesselt et al. (2010). The number of breakpoints was adjusted so as to obtain reasonable estimates of the trend changes mirroring the assumed underlying processes.

Antenna changes and other events influencing the vertical trend component were taken from the GPS log files for the respective stations. Some of the GPS stations examined here are located near tide gauges. Here, the trends from the tide gauges were compared with the GPS station height trends and sea level radar altimetry data from the TOPEX/Poseidon mission to separate the origin of the land movement trend 
signal where possible. All tide gauge data were retrieved from the Permanent Service for Mean Sea Level (PSMSL) (Woodworth and Player, 2003). Linear trends at tide gauges were determined using a standard linear model with ordinary least square adjustment. TOPEX/Poseidon sea level anomaly data were provided by Saskia Esselborn, GFZ. A seasonal component was extracted using the Loess algorithm from the STL package (Cleveland et al., 1990).

No atmospheric loading corrections were applied to the GPS data, except for BRAZ station, where the hydrological seasonal cycle was the main point of interest, and VAAS and MAR6 stations, for which also hydrological issues were reviewed. That is why the data of BRAZ, VAAS and MAR6 stations were corrected for the atmospheric loading using Green's functions as described in detail in Sect. 4.3.4.

While most stations show consistent, steady height trends, a minority demonstrate deviations caused by antenna or receiver changes. Following the recommendations by Blewitt and Lavallée (2002), stations with the total length of time series shorter than $2.5 \mathrm{yr}$ were not considered for the trend estimation. The vertical velocities of GPS stations located at tide gauges and some IGS stations computed by us are presented in Table 2. The seasonal component was removed from the time series before estimating the trend leading to small error estimates given in Tables 2-3 for our solution.

The time series of height changes of all GPS stations of GT1 solution are available at ftp://ftp.gfz-potsdam.de/pub/ home/ig/nana/GPS_station_heights/. In the following, a few interesting examples are treated in detail.

\subsection{Stations with prominent secular height trend}

\subsubsection{Plate tectonics: Neah Bay (NEAH), Canada}

Neah Bay, Washington, lies on the Juan de Fuca Strait in the Cascadia subduction zone, which is subject to crustal uplift as the North American plate is shifted over the Juan de Fuca plate. The GPS time series provides a secular trend of $3.96 \pm$ $0.02 \mathrm{~mm} \mathrm{yr}^{-1}\left(4.0 \pm 0.0 \mathrm{~mm} \mathrm{yr}^{-1}\right.$ with a seasonal signal removed), and the tide gauge measurements give a secular trend of $-1.8 \pm 0.1 \mathrm{~mm} \mathrm{yr}^{-1}$ (Fig. 7). This agrees well with the results of Verdonck (2006), who computed an estimated rate of land movement of $4.0 \pm 0.1 \mathrm{~mm} \mathrm{yr}^{-1}$. In his estimate, he subtracts a mean eustatic sea level rise $2.0 \mathrm{~mm} \mathrm{yr}^{-1}$ from the trend calculated from the tide gauge data $\left(-2.0 \mathrm{~mm} \mathrm{yr}^{-1}\right)$.

\subsubsection{Glacial isostatic adjustment: Fennoscandia (Skellefteå, Sweden (Furuogrund tide gauge), Mårtsbo, Sweden (Nedre Gavle tide gauge), and Vaasa, Finland (Vaasa tide gauge))}

These three stations, all located around the Gulf of Bothnia, are subject to land movement processes due to glacial isostatic adjustment (GIA). All stations show large secular up- lift rates with Skellefteå leading at $10.80 \pm 0.06 \mathrm{~mm} \mathrm{yr}^{-1}$, followed by Vaasa at $8.66 \pm 0.04 \mathrm{~mm} \mathrm{yr}^{-1}$ and Mårtsbo (7.62 \pm $0.01 \mathrm{~mm} \mathrm{yr}^{-1}$ ).

The GPS station Skellefteå lies in the vicinity of Furuogrund tide gauge (approximately $11 \mathrm{~km}$ distance). The tide gauge trend computed by us using full data and for the case, when the annual signal is removed, is $-8.1 \pm 0.2 \mathrm{~mm} \mathrm{yr}^{-1}$. So, the residual sea level rise from the sum of the tide gauge and vertical land movement trends is $2.7 \pm 0.3 \mathrm{~mm} \mathrm{yr}^{-1}$.

Nedre Gavle tide gauge situated at approximately $10 \mathrm{~km}$ distance from the GPS station MAR6 has a trend of $-6.0 \pm$ $0.2 \mathrm{mmyr}^{-1}$ (1896-1986) computed by us (Fig. 8, upper panel). Since the gauge stopped operating in 1986, there is no common period with the GPS station. The comparison with the land movement trend at Marrtsbo $\left(7.62 \pm 0.01 \mathrm{~mm} \mathrm{yr}^{-1}\right)$ indicates that a large part of the tide gauge signal can be explained by the land movement through GIA. Assuming that the GIA trend was constant over the last $100 \mathrm{yr}$, the residual sea level trend from the sum of the tide gauge relative sea level trend and land movement signals is $1.62 \pm 0.2 \mathrm{~mm} \mathrm{yr}^{-1}$.

Vaasa tide gauge trend estimated by us is $-7.6 \pm$ $0.2 \mathrm{~mm} \mathrm{yr}^{-1}$. The vertical land motion at the co-located GPS station VAAS with atmospheric loading correction applied is $8.6 \pm 0.2 \mathrm{mmyr}^{-1}$. So, the sum of the tide gauge trend and vertical land motion leads to the absolute sea level rise at Vaasa equal to $1.0 \pm 0.2 \mathrm{~mm} \mathrm{yr}^{-1}$.

Despite rather large distance $(>340 \mathrm{~km})$ between GPS stations Vaasa and Mårtsbo, the height time series for these stations are strongly correlated at $R^{2}=0.93$. When reduced for atmospheric loading, the correlation drops only marginally to $R^{2}=0.92$ (Fig. 8, lower panel). A large part of this is due to the trend. However, even the detrended time series score a $R^{2}=0.45$ correlation (Fig. 9, lower panel). The deviations between the two series consist mainly in an annual multiweek drift at Vaasa, which is caused by snow cover on the antenna. This figure demonstrates that the drift signals coincide with the periods of increased snow coverage in the area. The snow cover data were taken from Robert Dill's hydrological land surface discharge model (LSDM, Dill (2008)). Its influence on the correlation is checked by decomposing the detrended time series and determining the correlations for the respective components. When the linear trend is removed, the time series correlate at $R^{2}=0.73$ for the residual nonlinear trend, and $R^{2}=0.59$ for the full series with the nonlinear trend removed. Only the snow-dominated seasonal cycle shows no correlation at $R^{2}=-0.09$. It is worth to remark that the snow disturbance is correctly removed by the STL algorithm when removing the seasonal cycle. This example illustrates that hydrological errors occurring at a seasonal frequency can be easily and automatically removed when the seasonal cycle is calculated by taking the multi-year monthly mean, instead of fitting a sine. The tide gauge time series exhibit the same strong correlation, with $R^{2}=0.95$ for the full time series and $R^{2}=0.91$ for the detrended time series, in 
Table 2. Linear height trends at GPS stations. The table contains all stations from the TIGA reprocessing campaign with time series longer than $2.5 \mathrm{yr}$ from which a reasonable linear trend could be determined. Time series that showed large gaps were left out, as were stations with obvious jumps or trend changes. Some of these examples are treated in Sect. 4. A seasonal trend was removed for all stations. Atmospheric corrections were not applied. The station longitude and latitude are expressed in degrees, arc minutes and arc seconds. The beginning and the end of the time series are given as the GPS week number. The length of the time series is provided in years.

\begin{tabular}{|c|c|c|c|c|c|c|c|}
\hline Station & Name & Longitude & Latitude & Trend $\left(\mathrm{mm} \mathrm{yr}^{-1}\right)$ & Begin & End & Length \\
\hline 0194 & Tobishima, Sakata, Japan & 1393251.6 & 391108.0 & $0.90 \pm 0.03$ & 939 & 1459 & 10.0 \\
\hline ABER & Aberdeen, UK & 3575511.2 & 570838.4 & $1.69 \pm 0.02$ & 976 & 1459 & 9.3 \\
\hline AJAC & Ajaccio, France & 84545.4 & 415538.8 & $0.31 \pm 0.02$ & 1045 & 1446 & 7.7 \\
\hline ALAC & Alicante, Spain & 3593107.6 & 382020.1 & $0.23 \pm 0.01$ & 1021 & 1459 & 8.4 \\
\hline ALGO & Algonquin Park, Canada & 2815543.1 & 455720.9 & $3.47 \pm 0.02$ & 939 & 1459 & 10.0 \\
\hline ALIC & Alice Springs, Australia & 1335307.9 & -234012.4 & $0.38 \pm 0.03$ & 939 & 1459 & 10.0 \\
\hline ALME & Almeria, Spain & 3573226.0 & 365109.1 & $1.40 \pm 0.03$ & 1094 & 1459 & 7.0 \\
\hline ALRT & Alert, Canada & 2973934.3 & 822939.5 & $5.15 \pm 0.03$ & 1175 & 1459 & 5.5 \\
\hline ANDE & Andenes, Norway & 160805.3 & 691933.8 & $2.48 \pm 0.04$ & 1096 & 1424 & 6.3 \\
\hline ANDO & Andenes, Norway & 160030.6 & 691641.9 & $2.49 \pm 0.03$ & 939 & 1424 & 9.3 \\
\hline ANKR & Ankara, Turkey & 324530.5 & 395314.5 & $-0.73 \pm 0.05$ & 939 & 1455 & 9.9 \\
\hline ANTA & Antalya, Turkey & 303633.9 & 364942.6 & $-2.27 \pm 0.18$ & 1245 & 1455 & 4.0 \\
\hline AOML & Key Biscayne, FL, USA & 2795016.1 & 254404.9 & $0.50 \pm 0.03$ & 939 & 1265 & 6.3 \\
\hline AREQ & Arequipa, Peru & 2883025.9 & -162755.9 & $3.81 \pm 0.02$ & 1138 & 1459 & 6.2 \\
\hline ARTU & Arti Ekaterinburg, Russia & 583337.6 & 562547.4 & $0.90 \pm 0.04$ & 1021 & 1459 & 8.4 \\
\hline AUCK & Whangaparaoa No3, NZ & 1745003.8 & -363610.2 & $-1.55 \pm 0.04$ & 939 & 1459 & 10.0 \\
\hline BAHR & Manama, Bahrain & 503629.3 & 261232.9 & $0.53 \pm 0.02$ & 939 & 1459 & 10.0 \\
\hline BAIE & Baie Comeau, Canada & 2914412.0 & 491112.6 & $3.49 \pm 0.03$ & 1147 & 1459 & 6.0 \\
\hline BAKE & Baker Lake, Canada & 2635951.6 & 641904.2 & $10.82 \pm 0.04$ & 1147 & 1438 & 5.6 \\
\hline BILI & Bilibino, Russia & 1662616.7 & 680434.1 & $0.28 \pm 0.03$ & 1025 & 1459 & 8.3 \\
\hline BISH & Bishkek, Kyrgyzstan & 743539.1 & 425232.1 & $-1.42 \pm 0.05$ & 939 & 1359 & 8.1 \\
\hline BJFS & Beijing, Fangshan, China & 1155332.9 & 393631.0 & $3.47 \pm 0.05$ & 1032 & 1459 & 8.2 \\
\hline BOGT & Bogotá, Colombia & 2855508.6 & 43824.3 & $-44.21 \pm 0.19$ & 942 & 1459 & 9.9 \\
\hline BOR1 & Borowice, Poland & 170424.4 & 521637.0 & $-0.04 \pm 0.01$ & 1192 & 1459 & 5.1 \\
\hline BRAZ & Brasilia, Brazil & 3120719.7 & -155650.9 & $-0.18 \pm 0.05$ & 940 & 1459 & 10.0 \\
\hline BRMU & Bermuda, UK & 2951813.4 & 322213.4 & $-0.54 \pm 0.03$ & 939 & 1459 & 10.0 \\
\hline BRUS & Brussels, Belgium & 42133.2 & 504752.1 & $0.78 \pm 0.03$ & 939 & 1459 & 10.0 \\
\hline BUR1 & Burnie, Tasmania & 1455453.4 & -410300.2 & $-0.24 \pm 0.03$ & 1004 & 1412 & 7.8 \\
\hline CABL & Port Orford, OR, USA & 2352612.0 & 425010.0 & $-0.06 \pm 0.04$ & 1188 & 1459 & 5.2 \\
\hline CAGL & Cagliari, Italy & 85821.9 & 390809.3 & $-0.15 \pm 0.03$ & 939 & 1459 & 10.0 \\
\hline CANT & Santander, Spain & 3561207.0 & 432819.1 & $0.38 \pm 0.04$ & 1094 & 1459 & 7.0 \\
\hline CART & Cartagena, Colombia & 2842758.1 & 102328.8 & $-2.12 \pm 0.07$ & 1047 & 1456 & 7.9 \\
\hline CAS1 & Casey, Antarctica & 1103110.9 & -661700.1 & $-0.41 \pm 0.08$ & 1088 & 1453 & 7.0 \\
\hline CASC & Cascais, Portugal & 3503453.3 & 384136.3 & $0.19 \pm 0.03$ & 955 & 1459 & 9.7 \\
\hline $\mathrm{CCV} 3$ & Cape Canaveral, FL, USA & 2792717.2 & 282736.8 & $0.23 \pm 0.22$ & 969 & 1411 & 8.5 \\
\hline CEDU & Ceduna, Australia & 1334835.4 & -315200.0 & $-1.07 \pm 0.03$ & 944 & 1459 & 9.9 \\
\hline CFAG & Caucete, Argentina & 2914602.5 & -313607.8 & $-0.69 \pm 0.04$ & 939 & 1459 & 10.0 \\
\hline CHA1 & Charleston, SC, USA & 2800925.7 & 324527.2 & $-0.73 \pm 0.04$ & 988 & 1245 & 4.9 \\
\hline CHAT & Chatham Island, NZ & 1832603.0 & -435720.8 & $-0.37 \pm 0.02$ & 939 & 1459 & 10.0 \\
\hline CHUM & Chumysh, Kazakhstan & 744503.9 & 425954.6 & $0.40 \pm 0.02$ & 939 & 1459 & 10.0 \\
\hline CHUR & Churchill, Canada & 2655440.6 & 584532.7 & $9.67 \pm 0.03$ & 939 & 1459 & 10.0 \\
\hline CKIS & Rarotonga, Cook Islands, NZ & 2001157.8 & -211203.7 & $0.04 \pm 0.03$ & 1131 & 1459 & 6.3 \\
\hline $\mathrm{COCO}$ & Cocos Island, Australia & 965002.3 & -121118.0 & $-0.68 \pm 0.04$ & 939 & 1459 & 10.0 \\
\hline CONZ & Concepcion, Chile & 2865828.3 & -365037.5 & $0.39 \pm 0.11$ & 1170 & 1459 & 5.6 \\
\hline CRO1 & St. Croix, U.S. Virgin Isl. & 2952456.4 & 174524.8 & $-1.87 \pm 0.03$ & 939 & 1459 & 10.0 \\
\hline CSAR & Caesarea, Israel & 345324.7 & 322917.7 & $0.50 \pm 0.04$ & 1263 & 1459 & 3.8 \\
\hline DAEJ & Daejeon, South Korea & 1272228.1 & 362357.9 & $1.51 \pm 0.07$ & 1001 & 1459 & 8.8 \\
\hline DARW & Darwin, Australia & 1310757.9 & -125037.3 & $-0.17 \pm 0.07$ & 1242 & 1457 & 4.1 \\
\hline DAV1 & Davis, Antarctica & 775821.4 & -683438.4 & $-1.92 \pm 0.03$ & 1038 & 1459 & 8.1 \\
\hline DRAG & Metzoke Dragot, Israel & 352331.4 & 313535.5 & $3.15 \pm 0.03$ & 1033 & 1459 & 8.2 \\
\hline DRAO & Penticton, Canada & 2402230.1 & 491921.4 & $-0.56 \pm 0.03$ & 1188 & 1459 & 5.2 \\
\hline DUBO & Lac du Bonnet, Canada & 2640801.7 & 501531.7 & $2.13 \pm 0.06$ & 1288 & 1459 & 3.3 \\
\hline DUM1 & Dumont d'Urville, Antarctica & 1400007.0 & -663954.3 & $-2.11 \pm 0.02$ & 939 & 1459 & 10.0 \\
\hline DUNT & Dunedin, NZ & 1703745.9 & -454851.5 & $-1.63 \pm 0.02$ & 1029 & 1459 & 8.3 \\
\hline EIJS & Eijsden, Netherlands & 54101.0 & 504529.7 & $3.58 \pm 0.06$ & 939 & 1459 & 10.0 \\
\hline ESTI & Esteli, Nicaragua & 2733816.3 & 130558.3 & $5.06 \pm 0.06$ & 1061 & 1207 & 2.8 \\
\hline FAIR & Fairbanks, AK, USA & 2123002.7 & 645840.8 & $2.52 \pm 0.06$ & 1188 & 1459 & 5.2 \\
\hline
\end{tabular}


Table 2. Continued.

\begin{tabular}{|c|c|c|c|c|c|c|c|}
\hline Station & Name & Longitude & Latitude & Trend $\left(\mathrm{mm} \mathrm{yr}^{-1}\right)$ & Begin & End & Length \\
\hline FLIN & Flin Flon, Canada & 2580119.1 & 544332.1 & $3.53 \pm 0.03$ & 1288 & 1459 & 3.3 \\
\hline FORT & Fortaleza, Brazil & 3213427.8 & -35238.8 & $1.40 \pm 0.06$ & 1188 & 1370 & 3.5 \\
\hline FREE & Freeport, Bahamas & 2810026.8 & 264209.1 & $1.78 \pm 0.07$ & 1013 & 1137 & 2.4 \\
\hline FTS1 & Fort Stevens, OR, USA & 2360238.1 & 461217.6 & $2.82 \pm 0.02$ & 939 & 1459 & 10.0 \\
\hline GAL1 & Galveston, TX, USA & 2651547.5 & 291947.6 & $-4.76 \pm 0.05$ & 939 & 1226 & 5.5 \\
\hline GALA & Galapagos Isl., Ecuador & 2694147.0 & 04433.7 & $-2.59 \pm 0.09$ & 939 & 1192 & 4.9 \\
\hline GENO & Genova, Italy & 85516.1 & 442509.8 & $-0.48 \pm 0.04$ & 967 & 1459 & 9.5 \\
\hline GETI & Geting, Malaysia & 1020619.7 & 61334.3 & $3.39 \pm 0.02$ & 990 & 1186 & 3.8 \\
\hline GLPT & Gloucester Point, VA, USA & 2833002.0 & 371454.8 & $-2.61 \pm 0.03$ & 939 & 1385 & 8.6 \\
\hline GLSV & Kiev, Ukraine & 302948.2 & 502151.1 & $0.20 \pm 0.03$ & 949 & 1459 & 9.8 \\
\hline GODE & Greenbelt, MD, USA & 2831023.4 & 390118.2 & $-1.56 \pm 0.03$ & 939 & 1459 & 10.0 \\
\hline GOLD & Goldstone, CA, USA & 2430638.7 & 352530.6 & $0.25 \pm 0.09$ & 1088 & 1459 & 7.1 \\
\hline GOPE & Ondrejov, Czech Republic & 144708.2 & 495449.3 & $-1.44 \pm 0.04$ & 1192 & 1459 & 5.1 \\
\hline GRAS & Grasse, France & 65514.1 & 434517.1 & $0.59 \pm 0.01$ & 939 & 1459 & 10.0 \\
\hline GRAZ & Graz, Austria & 152936.5 & 470401.7 & $0.43 \pm 0.02$ & 939 & 1459 & 10.0 \\
\hline GUAM & Dededo, Guam & 1445206.1 & 133521.6 & $-0.01 \pm 0.04$ & 1138 & 1459 & 6.2 \\
\hline GUAT & Guatemala, Guatemala & 2692847.3 & 143525.5 & $1.04 \pm 0.06$ & 1072 & 1459 & 7.4 \\
\hline HELG & Helgoland Island, Germany & 75335.1 & 541028.1 & $1.04 \pm 0.02$ & 1035 & 1459 & 8.2 \\
\hline HILO & Hilo, Hawaii, USA & 2045650.3 & 194309.1 & $-1.66 \pm 0.02$ & 939 & 1454 & 9.9 \\
\hline HLFX & Halifax, Canada & 2962319.4 & 444100.8 & $-1.13 \pm 0.02$ & 1147 & 1459 & 6.0 \\
\hline HNLC & Honolulu, Hawaii, USA & 2020807.6 & 211811.8 & $-0.66 \pm 0.04$ & 939 & 1459 & 10.0 \\
\hline HNPT & Horn Point, Cambridge, MD, USA & 2835210.7 & 383519.7 & $-2.51 \pm 0.02$ & 1038 & 1459 & 8.1 \\
\hline HOB2 & Hobart, Australia & 1472619.4 & -424817.0 & $-0.53 \pm 0.04$ & 1038 & 1459 & 8.1 \\
\hline HOFN & Hoefn, Iceland & 3444807.5 & 641602.3 & $13.52 \pm 0.06$ & 1138 & 1459 & 6.2 \\
\hline HOLM & Holman, Canada & 2421419.5 & 704410.7 & $2.63 \pm 0.03$ & 1129 & 1459 & 6.3 \\
\hline HRAO & Hartebeesthoek, South Africa & 274113.1 & -255324.4 & $-0.72 \pm 0.02$ & 942 & 1457 & 9.9 \\
\hline IISC & Bangalore, India & 773413.3 & 130116.2 & $0.06 \pm 0.03$ & 939 & 1459 & 10.0 \\
\hline INEG $^{1}$ & Aguascalientes, Mexico & 2574256.9 & 215122.2 & $-87.66 \pm 0.28$ & 1038 & 1158 & 2.3 \\
\hline IRKT & Irkutsk, Russia & 1041858.5 & 521308.5 & $1.26 \pm 0.03$ & 939 & 1459 & 10.0 \\
\hline JOZE & Jozefoslaw, Poland & 210153.5 & 520550.2 & $1.00 \pm 0.01$ & 939 & 1459 & 10.0 \\
\hline JPLM & Pasadena, CA, USA & 2414936.4 & 341217.4 & $0.91 \pm 0.06$ & 939 & 1459 & 10.0 \\
\hline KARR & Karratha, Australia & 1170549.9 & -205853.1 & $0.55 \pm 0.04$ & 939 & 1459 & 10.0 \\
\hline KELS & Kelso, WA, USA & 2370614.2 & 460705.4 & $-0.53 \pm 0.02$ & 939 & 1444 & 9.7 \\
\hline KEN1 & Kenai, AK, USA & 2083859.3 & 604030.3 & $9.17 \pm 0.12$ & 939 & 1443 & 9.7 \\
\hline KERG & Kerguelen, Kerguelen Islands & 701519.9 & -492105.3 & $0.99 \pm 0.02$ & 1188 & 1459 & 5.2 \\
\hline KGNO & Koganei, Japan & 1392918.7 & 354224.4 & $0.83 \pm 0.02$ & 1128 & 1368 & 4.6 \\
\hline KIRI & Betio, Kiribati & 1725522.4 & 12116.5 & $-2.03 \pm 0.06$ & 1177 & 1459 & 5.4 \\
\hline KIRU & Kiruna, Sweden & 205806.4 & 675126.5 & $6.48 \pm 0.07$ & 939 & 1459 & 10.0 \\
\hline KIT3 & Kitab, Uzbekistan & 665307.6 & 390805.2 & $-0.95 \pm 0.05$ & 939 & 1459 & 10.0 \\
\hline KODK & Kodiak, AK, USA & 2072955.0 & 574406.4 & $8.82 \pm 0.10$ & 1039 & 1385 & 6.7 \\
\hline KOSG & Kootwijk, Netherlands & 54834.7 & 521042.3 & $-0.44 \pm 0.02$ & 939 & 1459 & 10.0 \\
\hline KOUR & Kourou, French Guyana & 3071138.5 & 51507.9 & $-0.32 \pm 0.09$ & 1088 & 1459 & 7.1 \\
\hline KSTU & Krasnoyarsk, Russia & 924737.8 & 555935.7 & $1.13 \pm 0.04$ & 949 & 1289 & 6.5 \\
\hline KUNM & Kunming, China & 1024749.9 & 250146.3 & $-0.08 \pm 0.06$ & 978 & 1459 & 9.3 \\
\hline KUUJ & Kuujjuarapik, Canada & 2821516.4 & 551642.1 & $11.52 \pm 0.06$ & 1173 & 1445 & 5.2 \\
\hline KWJ1 & Kwajalein, Marshall Islands & 1674348.9 & 84319.9 & $-1.63 \pm 0.14$ & 939 & 1176 & 4.6 \\
\hline KYW1 & Key West, FL, USA & 2782049.1 & 243456.2 & $-1.01 \pm 0.01$ & 939 & 1448 & 9.8 \\
\hline LAE1 & Lae, Papua New Guinea & 1465935.5 & -64025.3 & $-5.39 \pm 0.04$ & 1095 & 1384 & 5.6 \\
\hline LAGO & Lagos, Portugal & 3511953.8 & 370556.2 & $-0.76 \pm 0.02$ & 1056 & 1459 & 7.8 \\
\hline LAMP & Lampedusa, Italy & 123620.4 & 352959.2 & $0.42 \pm 0.02$ & 1002 & 1459 & 8.8 \\
\hline LAUT & Lautoka, Fiji & 1772647.7 & -173631.7 & $-0.19 \pm 0.11$ & 1141 & 1459 & 6.1 \\
\hline LHAS & Lhasa, Tibet, China & 910614.4 & 293926.4 & $0.82 \pm 0.03$ & 939 & 1412 & 9.1 \\
\hline LPGS & La Plata, Argentina & 3020403.7 & -345424.3 & $2.78 \pm 0.05$ & 939 & 1459 & 10.0 \\
\hline LROC & La Rochelle, France & 3584650.5 & 460932.2 & $0.29 \pm 0.03$ & 1141 & 1459 & 6.1 \\
\hline LYTT & Lyttelton, NZ & 1724320.0 & -433621.0 & $-0.95 \pm 0.02$ & 1037 & 1459 & 8.1 \\
\hline MAC1 & MacQuarie, Australia & 1585609.0 & -542958.3 & $-2.84 \pm 0.03$ & 939 & 1459 & 10.0 \\
\hline MADR & Madrid (Robledo), Spain & 3554501.2 & 402545.0 & $-0.22 \pm 0.08$ & 939 & 1459 & 10.0 \\
\hline MALI & Malindi, Kenya & 401139.8 & -25945.3 & $-0.87 \pm 0.11$ & 939 & 1459 & 10.0 \\
\hline MAR6 & Mårtsbo, Sweden & 171530.7 & 603542.5 & $7.62 \pm 0.01$ & 999 & 1459 & 8.8 \\
\hline MARS & Marseille, France & 52113.6 & 431643.6 & $0.33 \pm 0.02$ & 966 & 1459 & 9.5 \\
\hline
\end{tabular}


Table 2. Continued.

\begin{tabular}{|c|c|c|c|c|c|c|c|}
\hline Station & Name & Longitude & Latitude & Trend $\left(\mathrm{mm} \mathrm{yr}^{-1}\right)$ & Begin & End & Length \\
\hline MAS1 & Maspalomas, Canary Islands, Spain & 3442200.2 & 274549.5 & $-0.62 \pm 0.04$ & 1038 & 1459 & 8.1 \\
\hline MATE & Matera, Italy & 164216.0 & 403856.9 & $1.05 \pm 0.02$ & 939 & 1459 & 10.0 \\
\hline MAW1 & Mawson, Antarctica & 625214.6 & -673617.2 & $-0.95 \pm 0.01$ & 939 & 1459 & 10.0 \\
\hline MBAR & Mbarara, Uganda & 304416.4 & 03605.3 & $2.68 \pm 0.05$ & 1123 & 1455 & 6.4 \\
\hline MDO1 & McDonald, TX, USA & 2555906.0 & 304049.8 & $0.27 \pm 0.03$ & 939 & 1459 & 10.0 \\
\hline METS & Metsahovi, Finland & 242343.1 & 601302.9 & $4.70 \pm 0.01$ & 939 & 1459 & 10.0 \\
\hline MIL1 & Milwaukee, WI, USA & 2720641.6 & 430009.1 & $-3.40 \pm 0.02$ & 939 & 1401 & 8.9 \\
\hline MKEA & Mauna Kea, Hawaii, USA & 2043237.2 & 194804.9 & $-2.46 \pm 0.03$ & 939 & 1459 & 10.0 \\
\hline MOB1 & Mobile, AL, USA & 2715833.2 & 301339.1 & $-4.03 \pm 0.05$ & 939 & 1459 & 10.0 \\
\hline MPLA & Mar del Plata, Argentina & 3022807.7 & -380208.3 & $-2.26 \pm 0.14$ & 1187 & 1455 & 5.2 \\
\hline MQZG & McQueens Valley, NZ & 1723916.9 & -434209.8 & $-2.11 \pm 0.04$ & 1029 & 1459 & 8.3 \\
\hline NAIN & Nain, Canada & 2981840.6 & 563213.1 & $4.09 \pm 0.03$ & 1197 & 1459 & 5.0 \\
\hline NANO & Nanoose Bay, Canada & 2355448.7 & 491741.3 & $0.60 \pm 0.03$ & 939 & 1459 & 10.0 \\
\hline NCDK & Duck, NC, USA & 2841455.5 & 361055.4 & $-3.16 \pm 0.15$ & 1267 & 1417 & 2.9 \\
\hline NEAH & Neah Bay, WA, USA & 2352230.3 & 481752.3 & $3.96 \pm 0.02$ & 939 & 1459 & 10.0 \\
\hline NEIA & Cananeia, Brazil & 3120430.1 & -250112.9 & $0.49 \pm 0.01$ & 1172 & 1458 & 5.5 \\
\hline NEWL & Newlyn, UK & 3542726.0 & 500610.9 & $-0.17 \pm 0.02$ & 977 & 1458 & 9.3 \\
\hline NEWP & Newport, OR, USA & 2355617.2 & 443506.1 & $1.57 \pm 0.02$ & 947 & 1418 & 9.1 \\
\hline NICO & Nicosia, Cyprus & 332347.2 & 350827.6 & $0.80 \pm 0.04$ & 939 & 1459 & 10.0 \\
\hline NKLG & N'Koltang, Gabon & 94019.6 & 02114.1 & $-0.03 \pm 0.03$ & 1055 & 1458 & 7.8 \\
\hline NOUM & Noumea, New Caledonia & 1662436.7 & -221611.5 & $-3.06 \pm 0.05$ & 939 & 1419 & 9.2 \\
\hline NPLD & Teddington, UK & 3593937.3 & 512515.5 & $0.61 \pm 0.04$ & 1095 & 1458 & 7.0 \\
\hline NRC1 & Ottawa, Canada & 2842234.2 & 452715.0 & $3.49 \pm 0.01$ & 939 & 1459 & 10.0 \\
\hline NRIL & Norilsk, Russia & 882135.2 & 692142.6 & $2.24 \pm 0.04$ & 1079 & 1459 & 7.3 \\
\hline NSTG & North Shields, UK & 3583336.5 & 550026.7 & $0.70 \pm 0.02$ & 1213 & 1459 & 4.7 \\
\hline NVSK & Novosibirsk, Russia & 831407.6 & 545026.2 & $0.63 \pm 0.05$ & 1070 & 1459 & 7.5 \\
\hline NYA1 & Ny-Alesund, Norway & 115155.1 & 785546.4 & $8.54 \pm 0.04$ & 948 & 1459 & 9.8 \\
\hline NYAL & Ny-Alesund, Norway & 115154.3 & 785546.5 & $8.81 \pm 0.04$ & 945 & 1459 & 9.9 \\
\hline OBE2 & Oberpfaffenhofen 2 , Germany & 111647.5 & 480510.2 & $0.88 \pm 0.05$ & 1126 & 1457 & 6.4 \\
\hline OBER & Oberpfaffenhofen, Germany & 111647.5 & 480510.2 & $-0.67 \pm 0.10$ & 939 & 1115 & 3.4 \\
\hline ONSA & Onsala, Sweden & 115531.9 & 572343.1 & $1.90 \pm 0.05$ & 939 & 1459 & 10.0 \\
\hline OUS2 & Dunedin, NZ & 1703039.4 & -455210.1 & $-1.38 \pm 0.03$ & 1164 & 1459 & 5.7 \\
\hline $\mathrm{P} 102$ & Okushiri, Japan & 1392921.1 & 420443.6 & $-4.12 \pm 0.03$ & 1214 & 1459 & 4.7 \\
\hline P103 & Asamushi, Aomori, Japan & 1405133.1 & 405351.0 & $1.81 \pm 0.01$ & 1212 & 1459 & 4.8 \\
\hline P104 & Oga, Japan & 1394212.4 & 395631.6 & $3.66 \pm 0.02$ & 1221 & 1459 & 4.6 \\
\hline P108 & Aburatsubo, Miura, Japan & 1393655.9 & 350936.6 & $-1.71 \pm 0.02$ & 1212 & 1459 & 4.8 \\
\hline P109 & Ogi, Japan & 1381652.5 & 374853.1 & $2.46 \pm 0.02$ & 1221 & 1459 & 4.6 \\
\hline P110 & Kashiwazaki, Japan & 1383030.7 & 372123.6 & $-2.17 \pm 0.23$ & 1224 & 1458 & 4.5 \\
\hline $\mathrm{P} 112$ & Mikuni, Japan & 1360855.8 & 361516.5 & $-0.95 \pm 0.01$ & 1221 & 1459 & 4.6 \\
\hline P114 & Tago, Nishiizu, Japan & 1384551.1 & 344824.8 & $-2.34 \pm 0.01$ & 1222 & 1459 & 4.6 \\
\hline $\mathrm{P} 115$ & Yaizu, Japan & 1381938.7 & 345214.4 & $-2.92 \pm 0.06$ & 1222 & 1459 & 4.6 \\
\hline P116 & Onisaki, Tokoname, Japan & 1364925.8 & 345414.3 & $8.27 \pm 0.06$ & 1222 & 1459 & 4.6 \\
\hline P117 & Kainan, Japan & 1351129.5 & 340838.9 & $4.34 \pm 0.01$ & 1222 & 1459 & 4.6 \\
\hline P118 & Tajiri, Iwami, Japan & 1341857.4 & 353537.3 & $-0.43 \pm 0.01$ & 1223 & 1459 & 4.5 \\
\hline P119 & Susa, Japan & 1313616.7 & 343739.3 & $0.49 \pm 0.02$ & 1213 & 1459 & 4.7 \\
\hline P120 & Kure, Nakatosa, Japan & 1331436.6 & 332000.6 & $7.03 \pm 0.07$ & 1222 & 1459 & 4.6 \\
\hline P124 & Okinawa, Tinen, Japan & 1274928.8 & 261046.1 & $0.15 \pm 0.03$ & 1222 & 1459 & 4.6 \\
\hline P201 & Wakkanai, Japan & 1414107.2 & 452428.2 & $1.51 \pm 0.02$ & 1212 & 1459 & 4.8 \\
\hline P202 & Abashiri, Japan & 1441708.9 & 440109.9 & $1.95 \pm 0.02$ & 1221 & 1459 & 4.6 \\
\hline P203 & Kushiro, Japan & 1442217.0 & 425832.1 & $1.96 \pm 0.09$ & 1263 & 1459 & 3.8 \\
\hline $\mathrm{P} 204$ & Hakodate, Japan & 1404328.3 & 414654.0 & $1.29 \pm 0.03$ & 1214 & 1459 & 4.7 \\
\hline P206 & Mera, Tateyama, Japan & 1394929.7 & 345507.7 & $-1.12 \pm 0.00$ & 1223 & 1459 & 4.5 \\
\hline P209 & Hamada, Japan & 1320358.4 & 345350.3 & $-0.67 \pm 0.01$ & 1223 & 1459 & 4.5 \\
\hline $\mathrm{P} 211$ & Aburatsu, Nichinan, Japan & 1312433.5 & 313437.1 & $-0.68 \pm 0.05$ & 1220 & 1459 & 4.6 \\
\hline $\mathrm{P} 212$ & Naha, Japan & 1273954.7 & 261248.0 & $-0.18 \pm 0.01$ & 1222 & 1459 & 4.6 \\
\hline P213 & Chichijima, Ogasawara, Japan & 1421140.6 & 270538.2 & $0.10 \pm 0.08$ & 1215 & 1459 & 4.7 \\
\hline PALM & Palmer Station, Antarctica & 2955656.0 & -644630.3 & $3.91 \pm 0.06$ & 965 & 1446 & 9.3 \\
\hline PAPE & Papeete, Tahiti & 2102538.2 & -173159.1 & $-0.12 \pm 0.03$ & 1253 & 1459 & 4.0 \\
\hline PARC & Punta Arenas, Chile & 2890712.4 & -530813.0 & $-1.26 \pm 0.02$ & 990 & 1459 & 9.0 \\
\hline PBL1 & Point Blunt, CA, USA & 2373451.8 & 375111.0 & $-0.15 \pm 0.07$ & 939 & 1261 & 6.2 \\
\hline
\end{tabular}


Table 2. Continued.

\begin{tabular}{|c|c|c|c|c|c|c|c|}
\hline Station & Name & Longitude & Latitude & Trend $\left(\mathrm{mm} \mathrm{yr}^{-1}\right)$ & Begin & End & Length \\
\hline PDEL & Ponta Delgada, Azores, Portugal & 3342014.0 & 374451.9 & $-2.08 \pm 0.03$ & 1058 & 1459 & 7.7 \\
\hline PERT & Perth, Australia & 1155306.9 & -314807.1 & $-6.30 \pm 0.05$ & 939 & 1459 & 10.0 \\
\hline PETP & Petropavlovsk-Kamchatsky, Russia & 1583625.5 & 530400.2 & $-4.15 \pm 0.07$ & 978 & 1459 & 9.3 \\
\hline PGC5 & North Saanich, Canada & 2363255.9 & 483854.7 & $-1.36 \pm 0.16$ & 1315 & 1459 & 2.8 \\
\hline PIMO & Manila, Philippines & 1210439.8 & 143808.6 & $0.80 \pm 0.01$ & 1200 & 1459 & 5.0 \\
\hline PLO3 & Point Loma, CA, USA & 2424525.1 & 323955.5 & $-0.01 \pm 0.09$ & 939 & 1382 & 8.5 \\
\hline PLUZ & Las Palmas, Canary Islands, Spain & 3443532.6 & 280848.2 & $-1.05 \pm 0.06$ & 1265 & 1438 & 3.3 \\
\hline PNGM & Lombrum, Papua New Guinea & 1472157.6 & -20235.6 & $-1.64 \pm 0.16$ & 1164 & 1459 & 5.7 \\
\hline POHN & Pohnpei, Micronesia & 1581236.4 & 65735.8 & $-1.02 \pm 0.05$ & 1216 & 1459 & 4.7 \\
\hline POLV & Poltava, Ukraine & 343234.5 & 493609.4 & $-0.25 \pm 0.04$ & 1119 & 1459 & 6.5 \\
\hline POR4 & New Castle, NH, USA & 2891725.7 & 430415.7 & $0.88 \pm 0.10$ & 1056 & 1295 & 4.6 \\
\hline POTS & Potsdam, Germany & 130357.9 & 522245.5 & $-0.63 \pm 0.04$ & 939 & 1459 & 10.0 \\
\hline PRDS & Calgary, Canada & 2454223.4 & 505216.9 & $0.70 \pm 0.08$ & 939 & 1459 & 10.0 \\
\hline QAQ1 & Qaqortoq, Greenland & 3135708.1 & 604255.0 & $4.97 \pm 0.06$ & 1167 & 1459 & 5.6 \\
\hline RABT & Rabat, Morocco & 3530844.6 & 335953.2 & $-1.08 \pm 0.03$ & 1062 & 1459 & 7.6 \\
\hline RAMO & Mitzpe Ramon, Israel & 344547.3 & 303551.4 & $1.08 \pm 0.04$ & 961 & 1459 & 9.6 \\
\hline RED1 & Reedy Point, DE, USA & 2842548.1 & 393341.2 & $-2.39 \pm 0.07$ & 996 & 1424 & 8.2 \\
\hline REYK & Reykjavik, Iceland & 3380240.2 & 640819.6 & $-3.08 \pm 0.05$ & 939 & 1459 & 10.0 \\
\hline REYZ & Reykjavik, Iceland & 3380240.3 & 640819.7 & $-1.09 \pm 0.03$ & 1170 & 1445 & 5.3 \\
\hline RIGA & Riga, Latvia & 240331.6 & 565655.0 & $1.56 \pm 0.03$ & 999 & 1459 & 8.8 \\
\hline RIOG & Rio Grande, Argentina & 2921456.0 & -534707.7 & $2.99 \pm 0.05$ & 939 & 1416 & 9.2 \\
\hline RWSN & Rawson, Argentina & 2945333.9 & -431756.0 & $-0.01 \pm 0.02$ & 1046 & 1456 & 7.9 \\
\hline SAG1 & Saginaw Bay, MI, USA & 2760944.0 & 433743.1 & $-3.04 \pm 0.02$ & 939 & 1454 & 9.9 \\
\hline SANT & Santiago, Chile & 2891953.2 & -330901.0 & $3.17 \pm 0.03$ & 939 & 1459 & 10.0 \\
\hline $\mathrm{SCH} 2$ & Schefferville, Canada & 2931002.6 & 544955.5 & $10.88 \pm 0.03$ & 939 & 1459 & 10.0 \\
\hline SCUB & Santiago de Cuba, Cuba & 2841415.7 & 200043.4 & $0.12 \pm 0.07$ & 1050 & 1459 & 7.9 \\
\hline SEAT & Seattle, WA, USA & 2374125.9 & 473914.3 & $-0.20 \pm 0.03$ & 939 & 1459 & 10.0 \\
\hline SELD & Seldovia, AK, USA & 2081736.0 & 592644.6 & $10.21 \pm 0.08$ & 1085 & 1459 & 7.2 \\
\hline SFER & San Fernando, Spain & 3534739.7 & 362751.6 & $1.22 \pm 0.03$ & 991 & 1459 & 9.0 \\
\hline SHEE & Sheerness, UK & 04436.3 & 512644.5 & $-0.32 \pm 0.05$ & 939 & 1459 & 10.0 \\
\hline $\mathrm{SIO} 3$ & Scripps, CA, USA & 2424458.5 & 325152.9 & $2.54 \pm 0.07$ & 1038 & 1459 & 8.1 \\
\hline SKE0 & Skellefteå, Sweden & 210253.8 & 645245.1 & $10.80 \pm 0.06$ & 1232 & 1459 & 4.4 \\
\hline SOFI & Sofia, Bulgaria & 232341.0 & 423321.9 & $0.17 \pm 0.02$ & 939 & 1459 & 10.0 \\
\hline SOL1 & Solomons Island, MD, USA & 2833246.0 & 381908.0 & $0.19 \pm 0.34$ & 939 & 1437 & 9.6 \\
\hline STJO & St. John's, Canada & 3071920.1 & 473542.9 & $0.59 \pm 0.02$ & 939 & 1459 & 10.0 \\
\hline SUTH & Sutherland, South Africa & 204837.7 & -322248.8 & $-0.28 \pm 0.02$ & 953 & 1458 & 9.7 \\
\hline SUWN & Suwon-shi, South Korea & 1270315.3 & 371631.9 & $0.61 \pm 0.06$ & 939 & 1459 & 10.0 \\
\hline SYOG & Syowa, Antarctica & 393501.5 & -690025.0 & $0.64 \pm 0.04$ & 939 & 1459 & 10.0 \\
\hline TERS & Terschelling, Netherlands & 51309.8 & 532145.9 & $0.80 \pm 0.05$ & 939 & 1459 & 10.0 \\
\hline THTI & Tahiti, Tahiti & 2102336.8 & -173437.4 & $-0.89 \pm 0.03$ & 960 & 1459 & 9.6 \\
\hline THU3 & Thule Air Base, Greenland & 2911029.9 & 763213.4 & $5.05 \pm 0.04$ & 1167 & 1459 & 5.6 \\
\hline TID1 & Tidbinbilla, Australia & 1485848.0 & -352357.1 & $0.22 \pm 0.04$ & 939 & 1459 & 10.0 \\
\hline TID2 & Tidbinbilla, Australia & 1485848.0 & -352357.1 & $0.36 \pm 0.04$ & 939 & 1453 & 9.9 \\
\hline TIDB & Tidbinbilla, Australia & 1485848.0 & -352357.1 & $-0.93 \pm 0.04$ & 1088 & 1459 & 7.1 \\
\hline TIXI & Tixi, Russia & 1285159.1 & 713804.1 & $0.89 \pm 0.02$ & 978 & 1459 & 9.3 \\
\hline TLSE & Toulouse, France & 12851.2 & 433338.5 & $-0.11 \pm 0.04$ & 1095 & 1459 & 7.0 \\
\hline TONG & Nuku'alofa, Tonga & 1844914.8 & -210841.0 & $-1.07 \pm 0.06$ & 1154 & 1459 & 5.9 \\
\hline TORP & Torrance, CA, USA & 2414009.8 & 334752.1 & $-0.08 \pm 0.04$ & 939 & 1459 & 10.0 \\
\hline TOW2 & Townsville, Australia & 1470320.5 & -191609.4 & $-0.54 \pm 0.03$ & 939 & 1459 & 10.0 \\
\hline TRAB & Trabzon, Turkey & 394632.0 & 405941.0 & $0.38 \pm 0.02$ & 1039 & 1455 & 8.0 \\
\hline TRDS & Trondheim, Norway & 101909.0 & 632217.0 & $4.93 \pm 0.03$ & 1086 & 1459 & 7.2 \\
\hline TRO1 & Troms $\varnothing$, Norway & 185622.7 & 693945.8 & $3.50 \pm 0.05$ & 948 & 1459 & 9.8 \\
\hline TRON & Trondheim, Norway & 101909.0 & 632217.0 & $-9.50 \pm 0.79$ & 939 & 1050 & 2.1 \\
\hline TSEA & Anchorage, AK, USA & 2100618.1 & 611114.4 & $3.53 \pm 0.07$ & 1016 & 1459 & 8.5 \\
\hline TSKB & Tsukuba, Japan & 1400515.0 & 360620.5 & $-0.14 \pm 0.05$ & 939 & 1459 & 10.0 \\
\hline TUKT & Tuktoyaktuk, Canada & 2270020.3 & 692617.6 & $-0.01 \pm 0.02$ & 1233 & 1459 & 4.3 \\
\hline TUVA & Funafuti, Tuvalu & 1791147.6 & -83131.0 & $-0.01 \pm 0.05$ & 1142 & 1459 & 6.1 \\
\hline UCLU & Ucluelet, Canada & 2342730.1 & 485532.3 & $2.82 \pm 0.09$ & 1138 & 1459 & 6.2 \\
\hline ULAB & Ulaanbaatar, Mongolia & 1070308.4 & 475154.2 & $0.83 \pm 0.02$ & 1087 & 1459 & 7.2 \\
\hline UNB1 & Fredericton, Canada & 2932129.9 & 455700.8 & $0.61 \pm 0.09$ & 1123 & 1388 & 5.1 \\
\hline UNSA & Salta, Argentina & 2943532.5 & -244338.8 & $-0.87 \pm 0.06$ & 1199 & 1459 & 5.0 \\
\hline
\end{tabular}


Table 2. Continued.

\begin{tabular}{|c|c|c|c|c|c|c|c|}
\hline Station & Name & Longitude & Latitude & Trend $\left(\mathrm{mm} \mathrm{yr}^{-1}\right)$ & Begin & End & Length \\
\hline USNO & US Naval Obs., WA, USA & 2825601.6 & 385508.3 & $-1.67 \pm 0.02$ & 939 & 1459 & 10.0 \\
\hline UZHL & Uzhgorod, Ukraine & 221751.4 & 483755.1 & $0.14 \pm 0.04$ & 1016 & 1459 & 8.5 \\
\hline VAAS & Vaasa, Finland & 214614.3 & 625740.3 & $8.66 \pm 0.04$ & 999 & 1459 & 8.8 \\
\hline VALD & Val d'Or, Canada & 2822609.0 & 480549.4 & $8.04 \pm 0.04$ & 1148 & 1459 & 6.0 \\
\hline VALE & Valencia, Spain & 3593944.5 & 392851.0 & $-1.33 \pm 0.06$ & 1094 & 1459 & 7.0 \\
\hline VANU & Port Vila, Vanuatu & 1681854.5 & -174438.3 & $-2.87 \pm 0.06$ & 1183 & 1459 & 5.3 \\
\hline VARS & Vardø, Norway & 310152.3 & 702010.9 & $3.70 \pm 0.04$ & 1086 & 1459 & 7.2 \\
\hline VENE & Venice, Italy & 121955.1 & 452613.1 & $-1.04 \pm 0.05$ & 1138 & 1437 & 5.8 \\
\hline VESL & Vesleskarvet, Antarctica & 3570929.6 & -714025.7 & $0.01 \pm 0.10$ & 970 & 1459 & 9.4 \\
\hline VILL & Villafranca, Spain & 3560252.9 & 402636.9 & $-1.75 \pm 0.03$ & 939 & 1459 & 10.0 \\
\hline VIMS & Wachapreague, VA, USA & 2841846.8 & 373630.1 & $-3.13 \pm 0.02$ & 939 & 1459 & 10.0 \\
\hline VIS0 & Visby, Sweden & 182202.3 & 573913.9 & $3.14 \pm 0.03$ & 999 & 1459 & 8.8 \\
\hline VTIS & Los Angeles, CA, USA & 2414222.2 & 334245.5 & $0.46 \pm 0.07$ & 987 & 1459 & 9.1 \\
\hline WARN & Warnemuende, Germany & 120605.1 & 541011.2 & $0.48 \pm 0.05$ & 1205 & 1459 & 4.9 \\
\hline WGTN & Wellington, NZ & 1744821.2 & -411924.4 & $-2.64 \pm 0.02$ & 939 & 1459 & 10.0 \\
\hline WGTT & Wellington, NZ & 1744653.7 & -411725.6 & $-4.22 \pm 0.02$ & 1040 & 1459 & 8.1 \\
\hline WILL & Williams Lake, Canada & 2374955.9 & 521412.7 & $1.92 \pm 0.03$ & 939 & 1459 & 10.0 \\
\hline WIS1 & Wisconsin Point, WI, USA & 2675905.2 & 464218.2 & $-0.88 \pm 0.05$ & 939 & 1453 & 9.9 \\
\hline WLAD & Wladyslawowo, Poland & 182507.5 & 544748.3 & $0.26 \pm 0.03$ & 1215 & 1459 & 4.7 \\
\hline WSRT & Westerbork, NL & 63616.2 & 525452.6 & $-0.60 \pm 0.02$ & 939 & 1459 & 10.0 \\
\hline WTZR & Wettzell, Germany & 125244.1 & 490839.1 & $-0.08 \pm 0.02$ & 939 & 1459 & 10.0 \\
\hline WUHN & Wuhan, China & 1142126.1 & 303154.0 & $4.66 \pm 0.09$ & 939 & 1459 & 10.0 \\
\hline YAKT & Yakutsk, Russia & 1294049.1 & 620151.5 & $-5.44 \pm 0.34$ & 992 & 1459 & 9.0 \\
\hline YAR1 & Yarragadee, Australia & 1152049.1 & -290247.6 & $-1.31 \pm 0.07$ & 939 & 1166 & 4.4 \\
\hline YELL & Yellowknife, Canada & 2453109.5 & 622851.2 & $6.20 \pm 0.02$ & 939 & 1459 & 10.0 \\
\hline YSSK & Yuzhno-Sakhalinsk, Russia & 1424300.2 & 470147.0 & $1.15 \pm 0.02$ & 1020 & 1459 & 8.4 \\
\hline ZECK & Zelenchukskaya, Russia & 413354.2 & 434718.2 & $1.83 \pm 0.02$ & 939 & 1459 & 10.0 \\
\hline ZIMM & Zimmerwald, $\mathrm{CH}$ & 72755.0 & 465237.6 & $1.67 \pm 0.03$ & 939 & 1459 & 10.0 \\
\hline
\end{tabular}

${ }^{1}$ Subsidence at Aguascalientes (INEG) has been treated in Esquivel et al. (2006)

Table 3. Comparison of the vertical velocities $\left(\mathrm{mmyr}^{-1}\right)$ of GPS stations common in our and three recent solutions.

\begin{tabular}{lrrrr}
\hline Station & Our solution & $\begin{array}{r}\text { Santamaría-Gómez et al. (2012) } \\
\text { solution }\end{array}$ & $\begin{array}{r}\text { Bouin and Wöppelmann (2010) } \\
\text { solution }\end{array}$ & $\begin{array}{r}\text { Zhang et al. (2008) } \\
\text { solution }\end{array}$ \\
\hline ALRT & $5.15 \pm 0.03$ & $6.43 \pm 0.44$ & $5.46 \pm 1.64$ & $8.96 \pm 0.05$ \\
BAHR & $0.53 \pm 0.02$ & $0.21 \pm 0.17$ & $0.93 \pm 0.72$ & $0.28 \pm 0.01$ \\
CHUR & $9.67 \pm 0.03$ & $10.36 \pm 0.10$ & $10.77 \pm 0.72$ & $10.80 \pm 0.04$ \\
FTS1 & $2.82 \pm 0.02$ & $2.13 \pm 0.34$ & $3.00 \pm 0.73$ & $-0.89 \pm 0.03$ \\
MAW1 & $-0.95 \pm 0.01$ & $-0.11 \pm 0.21$ & $-0.35 \pm 0.72$ & $3.20 \pm 0.02$ \\
MOB1 & $-4.03 \pm 0.05$ & $-3.05 \pm 0.39$ & $-3.58 \pm 0.76$ & $-2.45 \pm 0.04$ \\
NANO & $0.60 \pm 0.03$ & $0.83 \pm 0.18$ & $1.84 \pm 0.80$ & $0.46 \pm 0.01$ \\
NEAH & $3.96 \pm 0.02$ & $2.63 \pm 0.25$ & $3.82 \pm 0.82$ & $2.93 \pm 0.02$ \\
NEWP & $1.57 \pm 0.02$ & $0.78 \pm 0.35$ & $1.61 \pm 0.77$ & $1.34 \pm 0.01$ \\
NOUM & $-3.06 \pm 0.05$ & $0.63 \pm 0.51$ & $-2.68 \pm 0.80$ & $-0.05 \pm 0.02$ \\
NYAL & $8.81 \pm 0.04$ & $8.27 \pm 0.26$ & $8.19 \pm 0.73$ & $8.32 \pm 0.01$ \\
PARC & $-1.26 \pm 0.02$ & $-0.15 \pm 0.18$ & $-1.39 \pm 0.87$ & $0.66 \pm 0.05$ \\
PERT & $-6.30 \pm 0.05$ & $-2.98 \pm 0.34$ & $-5.21 \pm 0.73$ & $-3.88 \pm 0.02$ \\
PETP & $-4.15 \pm 0.07$ & $-1.91 \pm 0.45$ & $-2.70 \pm 0.88$ & $-3.91 \pm 0.02$ \\
SEAT & $-0.20 \pm 0.07$ & $-1.34 \pm 0.23$ & $0.14 \pm 0.81$ & $-0.98 \pm 0.02$ \\
SFER & $1.22 \pm 0.03$ & $-0.15 \pm 0.18$ & $1.60 \pm 0.89$ & $2.00 \pm 0.02$ \\
STJO & $0.59 \pm 0.02$ & $-0.10 \pm 0.15$ & $-0.22 \pm 0.72$ & $-0.30 \pm 0.01$ \\
SYOG & $0.64 \pm 0.04$ & $2.04 \pm 0.44$ & $2.75 \pm 0.80$ & $3.45 \pm 0.02$ \\
TRO1 & $3.50 \pm 0.05$ & $3.12 \pm 0.20$ & $3.43 \pm 0.82$ & $3.95 \pm 0.02$ \\
WGTT & $-4.22 \pm 0.02$ & $-2.83 \pm 0.33$ & $-3.94 \pm 1.04$ & $-1.64 \pm 0.03$ \\
\hline
\end{tabular}



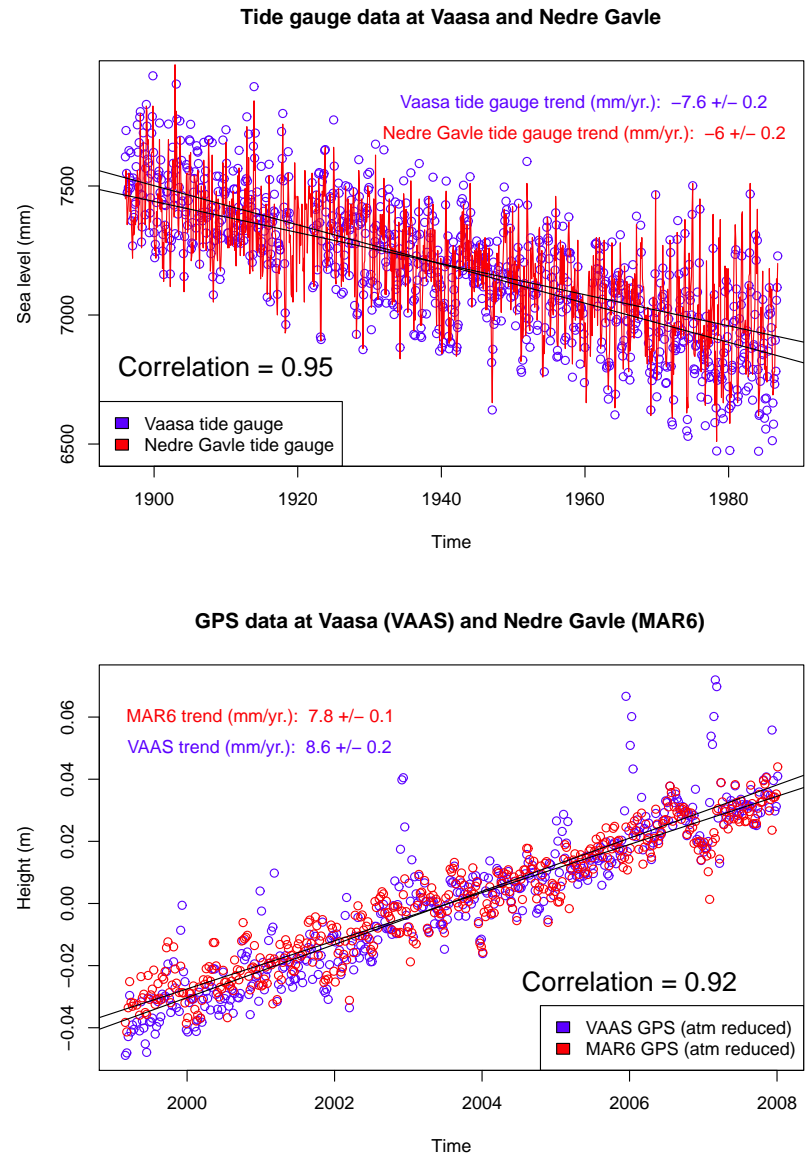

Fig. 8. The correlation of the height time series of two tide gauges Vaasa and Nedre Gavle (the upper panel) situated on the opposite sides of the Gulf of Bothnia at approximately $340 \mathrm{~km}$ distance. The correlation of the height time series of their co-located GPS stations, VAAS and MAR6 (with atmospheric loading correction applied, the lower panel).

both cases for the common period (1896-1986) (Fig. 9, upper panel).

\subsubsection{Glacial isostatic adjustment: Canada (Churchill, CHUR, and Kuujjuarapik, KUUJ)}

Both Churchill and Kuujjuarapik lie in the Hudson Bay, which is also subject to the glacial isostatic adjustment. Both GPS stations show strong secular uplift trends: $9.67 \pm$ $0.03 \mathrm{~mm} \mathrm{yr}^{-1}$ (CHUR) and $11.52 \pm 0.06 \mathrm{mmyr}^{-1}$ (KUUJ) (Fig. 10, middle panel). Despite their distance $(1067 \mathrm{~km})$, the two GPS stations display significant correlation $(R=0.92)$ of their height time series, also of the detrended ones $(R=$ 0.47, Fig. 10, lower panel). The secular trend in the GPS data is nicely visible also in the Churchill tide gauge data. For 1940-2009, the Churchill tide gauge yields a trend of $-9.7 \pm 0.1 \mathrm{~mm} \mathrm{yr}^{-1}\left(-9.54 \pm 0.2 \mathrm{mmyr}^{-1}\right.$ with a seasonal component removed). It is clear even from the visual inspection of the Churchill tide gauge (Fig. 10, upper panel) that the overall negative regional sea level trend has flattened considerably since the beginning of the 1990s. For the 1998-2007, the trend at Churchill yields $-2.8 \pm 3.5 \mathrm{~mm} \mathrm{yr}^{-1}$. Removing the seasonal signal reduces the standard error and results in a substantially larger trend estimate of $-5.18 \pm 0.97 \mathrm{~mm} \mathrm{yr}^{-1}$. This means that, for 1998-2007, the full data trend is around two-thirds smaller than for the whole period of coverage, and about half when the seasonal signal is removed. An explanation for this behaviour can be found in Déry et al. (2011), who mention that there is a notable positive trend in river discharge into Hudson Bay, starting around the 1990s and continuing until 2008, the end of their time series. At the same time, there is a shift in the seasonality of river discharge into the bay. With a positive trend in winter and negative trend in summer streamflow, the time series variance is expected to increase. This explains the large impact of the seasonal signal on the trend for the 1998-2007 time series. The tide gauge signal, which has long been dominated by GIA, is apparently now influenced much more strongly by the changing hydrological processes in Hudson Bay.

\subsubsection{Bogotá (BOGT), Colombia}

This station is a peculiar example. It is located near the building of the Instituto Geográfico Agustín Codazzi (IGAC) in Bogotá and has been continuously subsiding during the past years. The station was treated in Kaniuth et al. (2001) together with another station, BOGA, which is positioned on the top of the IGAC building. In their treatment, the authors suggest that construction work may have worsened the subsidence processes caused by sedimentation in the area. The vertical trend at BOGT has almost doubled since it was first estimated in their 2001 paper $\left(-25.2 \pm 1.4 \mathrm{~mm} \mathrm{yr}^{-1}\right)$, now reaching the alarming rate of $-44.21 \pm 0.19 \mathrm{~mm} \mathrm{yr}^{-1}$ (Fig. 11). Obviously, the station cannot be used for any purposes within the TIGA framework.

\subsection{Stations with height trend changes}

\subsubsection{Trend changes in GPS station data: Arequipa (AREQ), Peru}

Arequipa lies in southwestern Peru, in the subduction zone where the oceanic Nazca plate is subducted under the South American plate along the Peru-Chile trench. It is an interesting example of a trend change, as the station recorded the 2001 Arequipa-Camana-Tacna area (M:8.4W) earthquake (Utsu, 1990) and its effects on land movement. The trend change is visible in the time series (Fig. 12) from GPS week 1120 (25 June 2001), two days after the date of the earthquake on 23 June 2001. Before the event, the trend was $-2.6 \pm 0.5 \mathrm{mmyr}^{-1}$, changing direction to a positive $4.2 \pm 0.1 \mathrm{mmyr}^{-1}$ uplift trend afterwards. From 2005 on, a slight trend reduction can be observed. This is consistent 

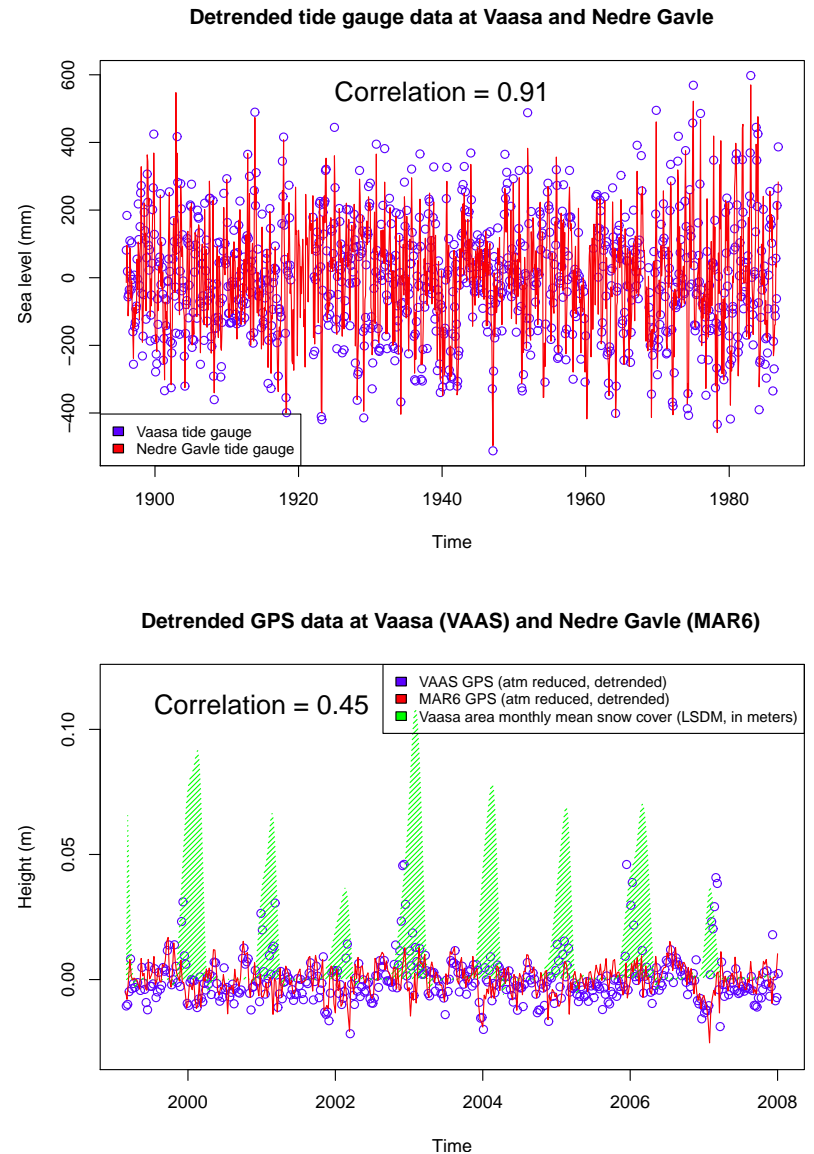

Fig. 9. The upper panel: the detrended sea level time series for tide gauges Vaasa and Nedre Gavle for the common period (18961986). The lower panel: the detrended height time series for the co-located GPS stations Vaasa (VAAS) and Nedre Gavle (MAR6). The green shading depicts the modelled mean snow cover at the four neighbouring grid points surrounding the station according to the LSDM hydrology model.

with exponential behaviour that can be expected for a postseismic event.

\subsubsection{Trend change in tide gauge data: Kushimoto (P208), Japan}

Kushimoto (GPS station P208) is located at the southern tip of the Kii Peninsula, Wakayama prefecture. It is situated within the Nankai trough subduction zone and was affected, among others, by the 1946 Nankai earthquake $(M=8)$ (Utsu, 1990). Sato et al. (1998) mention that, prior to the 1944 earthquake, the southern part of the peninsula is supposed to have subsided with a constant rate of $5-6 \mathrm{~mm} \mathrm{yr}^{-1}$. The Kushimoto tide gauge record indicates a clear trend change coinciding with the minor $(M=5.6)$ Wakayama prefecture earthquake of 9 May 1987 (Utsu, 1990). The relative sea level trend from the tide gauge before the earthquake is a moderate $+0.6 \pm 0.2 \mathrm{~mm} \mathrm{yr}^{-1}$, increasing by almost ten times to
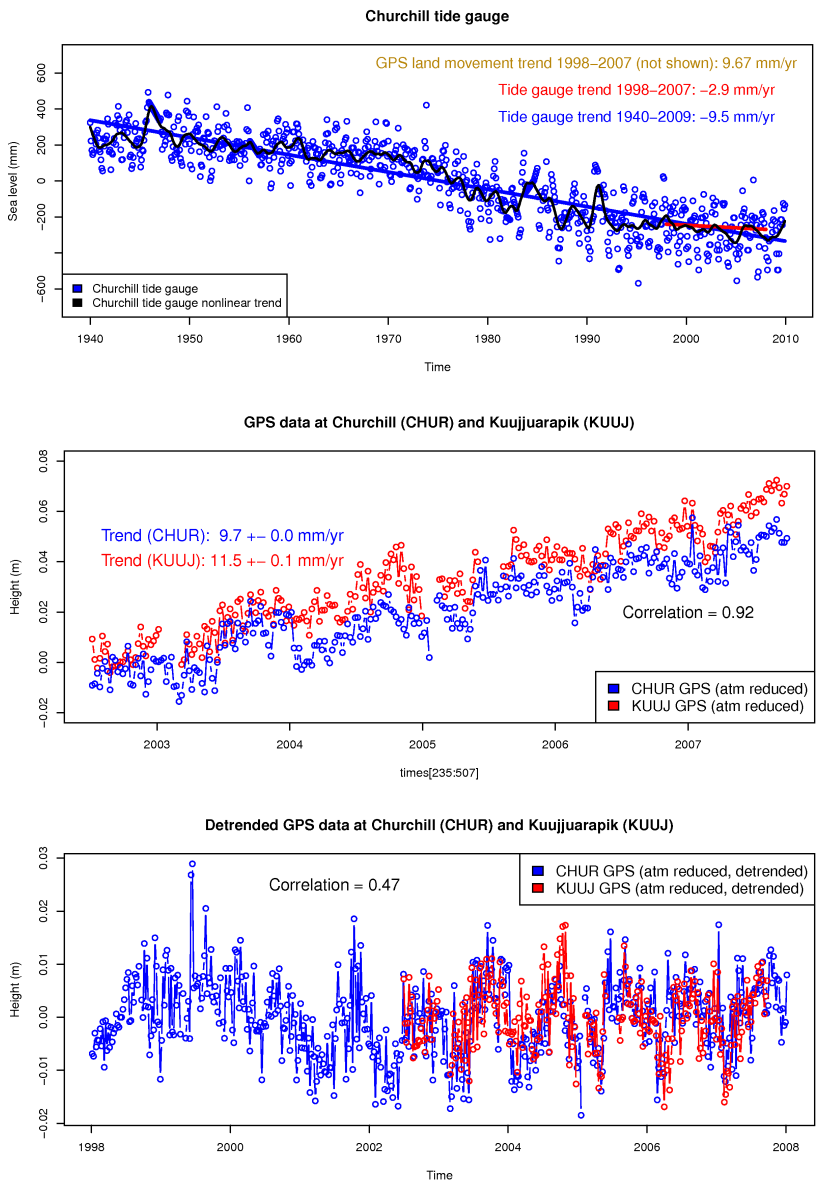

Fig. 10. The upper panel: sea level time series at tide gauge at Churchill; the middle panel: height time series and trends at GPS stations CHUR (Churchill) and KUUJ (Kuujjuarapik); the lower panel: detrended height time series at these GPS stations.

$+6.5 \pm 0.2 \mathrm{mmyr}^{-1}$ after the earthquake. The vertical land movement trend determined from the GPS station P208 over the 2005-2010 period is $-6.3 \pm 0.1 \mathrm{~mm} \mathrm{yr}^{-1}$. A comparison with the TOPEX/Poseidon (1994-2001) sea level anomaly trend $\left(-1.8 \pm 1.7 \mathrm{~mm} \mathrm{yr}^{-1}\right)$ strengthens the assumption that the major part of the tide gauge trend is caused by the land movement (Fig. 13).

Apparently, after the 1987 earthquake, the southern part of the Kii peninsula resumed its original subsiding motion. Still, (Isoda et al., 2004) remarked that the Kushimoto tide gauge is located on the (uplifting) Eurasia plate. Emery and Aubrey (1991) mentioned submergence caused by groundwater extraction as another possible cause for subsidence.

\subsubsection{Trend change in tide gauge and GPS data: Aburatsu (P211), Japan}

Another example of a trend change is Aburatsu tide gauge located at Nichinan, Miyazaki prefecture, Japan. One trend 


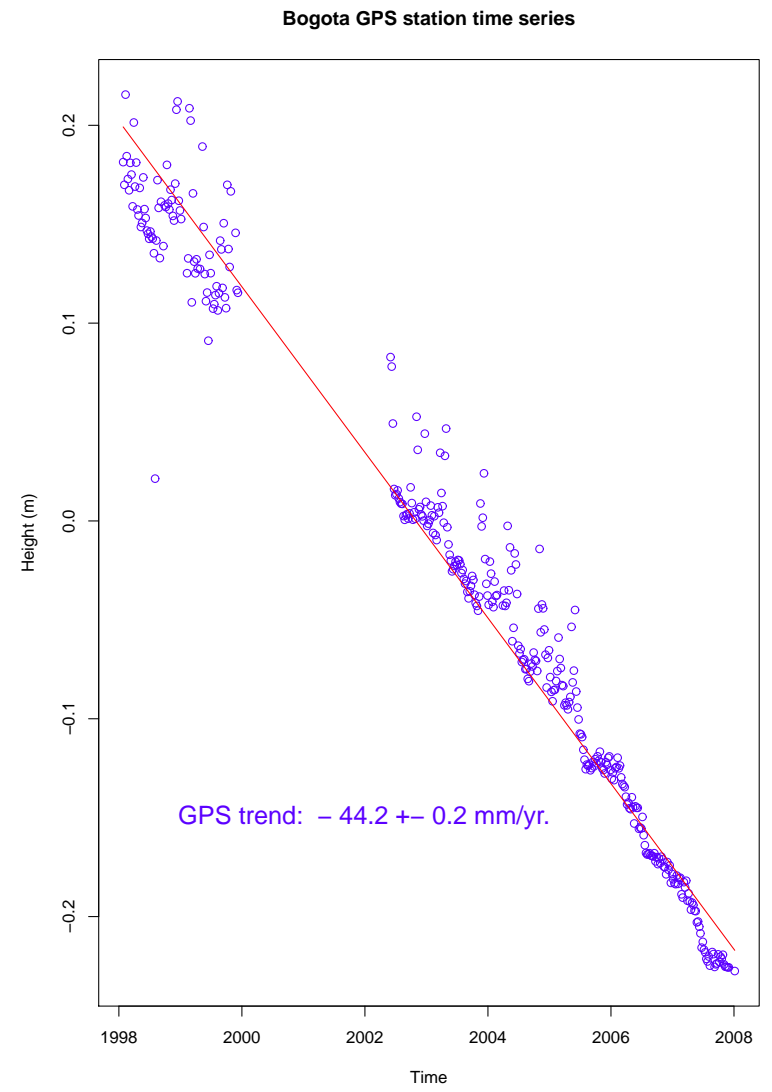

Fig. 11. GPS station Bogotà (BOGT) exhibits a significant secular height trend of $-44.2 \pm 0.2 \mathrm{~mm} \mathrm{yr}^{-1}$.

change (Fig. 14, upper panel) occurs in the aftermath of the July, 1970 Miyazaki prefecture earthquake $(M=6.7)$. Another trend change occurs in April 1984 and coincides with the Kagoshima prefecture earthquake of 28 April 1986 $(\mathrm{M}=4.4)$ (Utsu, 1990).

An analysis of the height time series for the co-located GPS station P211 shows (Fig. 14, lower panel) a trend change from GPS week 1342 (26 September 2005). Before this (May 2003-May 2005), the land movement trend from the GPS time series with a value of $-3.3 \pm 0.6 \mathrm{~mm} \mathrm{yr}^{-1}$ explains a large part of the sea level rise trend of $3.5 \pm 1.0 \mathrm{~mm} \mathrm{yr}^{-1}$ computed for the time after the last trend change (from April 1987). After the 2005 trend change, the land movement switches sign to $2.1 \pm 0.7 \mathrm{~mm} \mathrm{yr}^{-1}$. Drops in the time series as depicted in Fig. 14 clearly mark the impact of the Oita 2006 (12 June, $M=6.2$ ) and 2007 (6 June, $M=4.9$ ) earthquakes. The impact of the 2005 Fukuoka earthquakes (Utsu, 1990) (20 and 22 March, 20 April, and 5 May) is less clear, but the trend change begins a few weeks after the earthquake.

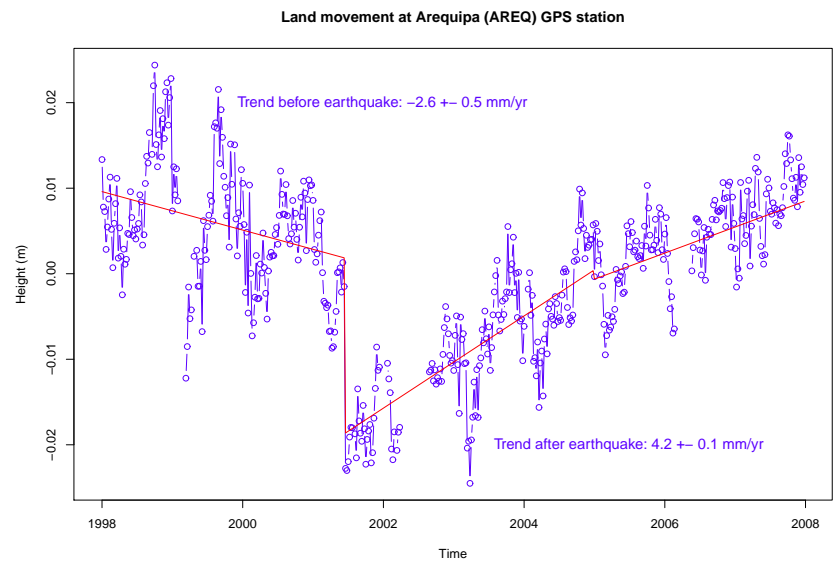

Fig. 12. The height trend change at GPS station Arequipa (AREQ) from $-2.6 \pm 0.5 \mathrm{~mm} \mathrm{yr}^{-1}$ to $+4.2 \pm 0.1 \mathrm{~mm} \mathrm{yr}^{-1}$ caused by the 2001 Arequipa-Camana-Tacna area earthquake.

\subsubsection{Variations caused by hydrological processes: Brasilia (BRAZ), Brazil}

The time series of this GPS station (Fig. 15) situated in the Roncador Ecological Reserve, approximately $35 \mathrm{~km}$ south of Brasilia, exhibits strong annual variations from hydrological effects. Van Dam et al. (2001) already mention BRAZ as an example for strong annual variations due to hydrological water loading, although, at the time of publication, there was only a short time series available.

To separate the atmospheric pressure loading effects from the hydrological variations, a pressure correction was applied following van Dam et al. (1994). NCEP 6-hourly surface pressure data were convolved with Farrell's Green's functions using the programme provided on Tonie van Dam's website (van Dam, 2010) and the technique outlined in van Dam et al. (1994).

From 2006 on, a strong negative trend is visible in the GPS time series. This decrease can be also found in GRACE data (Kurtenbach et al., 2009). For the comparison, the GRACE data were resampled to monthly values and filtered, then converted to equivalent water height. The gridded product was provided by Henryk Dobslaw, GFZ. For a qualitative comparison, the GRACE water column data are scaled by factor 150 in Fig. 15. The comparison with water mass data from the LSDM model (Dill, 2008) also shows a strong decrease in water column height. The cause of the subsidence is apparently the drought that hit this region in late summer and fall of 2006 (EM-DAT, 2011).

\subsection{Stations with zero height trend}

Tuktoyaktuk (TUKT) station in northwestern Canada is an example for a site with a height trend very close to zero $\left(-0.01 \pm 0.02 \mathrm{~mm} \mathrm{yr}^{-1}\right)$. Located close to the Alaskan border, the station is apparently affected neither by the positive 

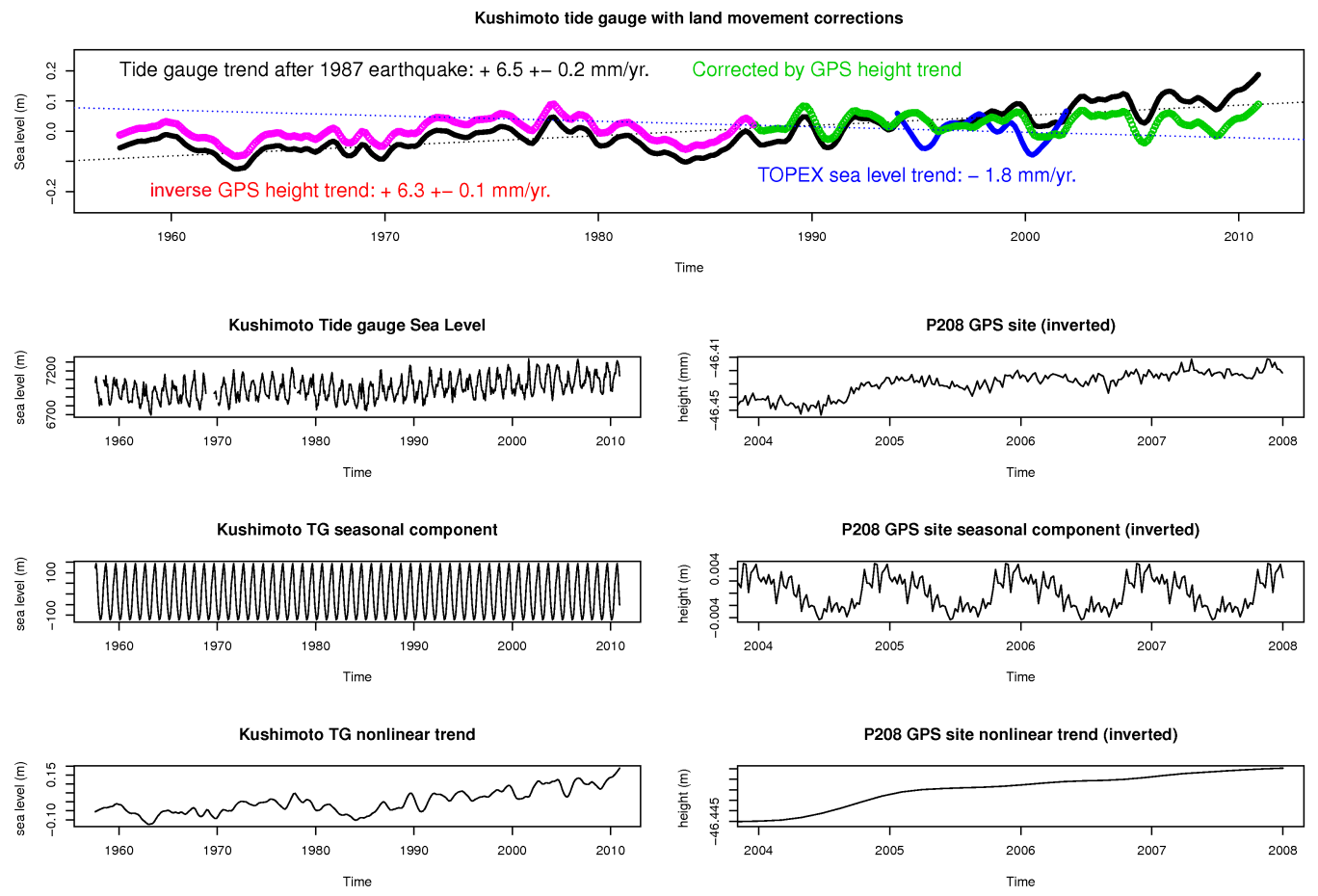

Fig. 13. The apparent sea level rise $\left(+6.5 \pm 0.2 \mathrm{~mm} \mathrm{yr}^{-1}\right)$ at Kushimoto tide gauge after the 1987 Wakayama prefecture earthquake and its comparison with the height trend $\left(-6.3 \pm 0.1 \mathrm{~mm} \mathrm{yr}^{-1}\right)$ at the co-located GPS station P208.

GIA signal centered on Eastern Canada, nor by the land movement signals caused by the recent ice loss (Larsen et al., 2004) commonly found in south Alaska. The moderate length of the time series ( $4.3 \mathrm{yr})$, however, makes this assessment a preliminary one. The seasonal cycle produces a spurious trend $\left(0.5 \pm 0.3 \mathrm{~mm} \mathrm{yr}^{-1}\right)$, if not removed (Fig. 16).

Some more GPS stations with longer time series demonstrate the height trend being very close to zero. These are, for example, GPS station CKIS (Rarotonga, Cook Islands, in free association with New Zealand) with the height trend of $0.04 \pm 0.03 \mathrm{~mm} \mathrm{yr}^{-1}$ obtained over the $6.3 \mathrm{yr}$ time series; PLO3 (Point Loma, CA, USA) for which the vertical velocity of $-0.01 \pm 0.09 \mathrm{~mm} \mathrm{yr}^{-1}$ was computed at the 8.5 -yr time interval; RWSN (Rawson, Argentina) with the height trend of $-0.01 \pm 0.02 \mathrm{~mm} \mathrm{yr}^{-1}$ calculated at the 7.9-yr time span; TUVA (Funafuti, Tuvalu) for which the vertical velocity of $-0.01 \pm 0.05 \mathrm{~mm} \mathrm{yr}^{-1}$ was estimated using the 6.1 -yr time series and some other stations.

\section{Discussion}

The linear height trends obtained in this work have been compared with previous solutions. Thus, our solution (Table 2) contains linear height trends additionally for the following 38 GPS stations not present in the previous GFZ solution (Zhang et al., 2008) due to the inclusion of GPS data from these stations located in Brazil, Canada, Iceland, Israel, Japan, Norway, Poland, Spain, Sweden, Tasmania, Turkey, the USA and on Tahiti in the GT1 solution: 0194, ANDE, ANDO, ANTA, BUR1, CSAR, NCDK, NEIA, P102, P103, P104, P108, P109, P110, P112, P114, P115, P116, P117, P118, P119, P120, P124, P201, P202, P203, P204, P206, P209, P211, P212, P213, PAPE, PLUZ, REYZ, SKE0, TUKT, VTIS, WLAD. This is a notable contribution to the densification of the GPS station network processed.

We have compared the vertical velocities of our solution with the previous GFZ solution (Zhang et al., 2008) obtained by processing GPS data from a global network of 370 GPS stations from January 1994 till December 2006 and containing the vertical velocities of 335 stations aligned to ITRF2000 (Altamimi et al., 2002) and two solutions of Université de La Rochelle: ULR3 solution (Bouin and Wöppelmann, 2010) derived using GPS data from a global network of 227 stations from January 1997 to November 2006 and containing the vertical velocities of 180 stations expressed in ITRF2005 and ULR5 solution (SantamaríaGómez et al., 2012) obtained by processing GPS data from a global network of 420 stations from January 1995 to December 2010 and providing the vertical velocities for 326 stations given in ITRF2008. Both ULR solutions use IGS absolute phase centre corrections for both tracking and transmitting antennas, as our (GT1) solution does, whereas the previous GFZ solution was computed using relative phase 

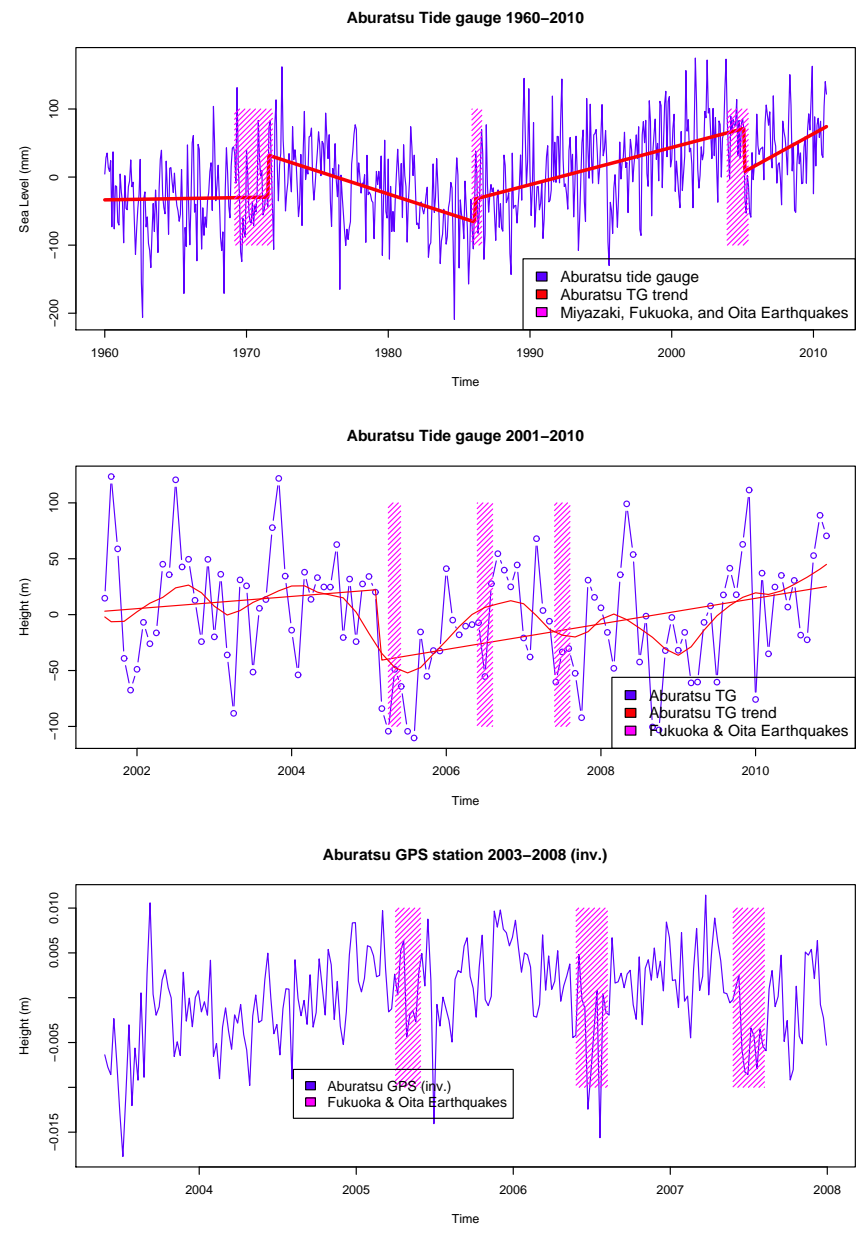

Fig. 14. The impact of earthquakes on Aburatsu tide gauge and colocated GPS station P211 height time series: Aburatsu tide gauge sea level time series (1960-2010, the upper panel), a close-up of the tide gauge time series for 2001-2010 (the middle panel) and inverted height time series at the GPS station P211 (2003-2008, the lower panel). The pink shading marks the interval for possible breakpoints detected by the BFAST algorithm caused by the 2005 Fukuoka earthquakes and the 2006 and 2007 Oita earthquakes.

centre corrections and some obsolete models. The comparison shows that the discrepancies in the vertical velocities are below $1 \mathrm{~mm} \mathrm{yr}^{-1}$ for 106 of 142 common stations (i.e. about 75 per cent) for GT1 and ULR3 solutions, for 120 of 179 common stations (i.e. about 67 per cent) for GT1 and ULR5 solutions, whereas only for 101 of 224 common stations (i.e. about 45 per cent) for GT1 and Zhang et al. (2008) solutions due to the use of some obsolete models, in particular, relative phase centre corrections in the previous GFZ solution. The vertical velocities of GPS stations common in our and three recent solutions are provided in Table 3 .

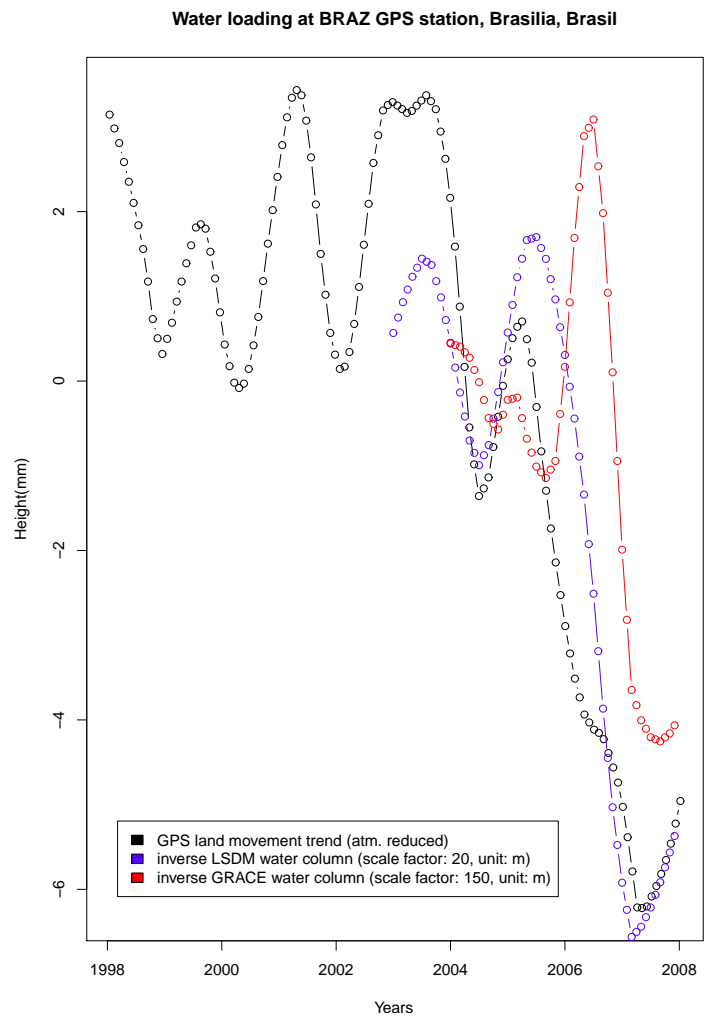

Fig. 15. A qualitative comparison of three different signals showing the 2006 drought in Brazil: height time series of GPS station Brasilia (BRAZ), inverse water storage mass and inverse hydrological loading from GRACE data. The nonlinear trend (seasonal signal and noise removed) is depicted in all three time series. Different scale factors are used for three plots.

\section{Conclusions}

We have reprocessed GPS data from a global network of 403 GPS stations over the 10-yr time span (1998-2007) using new models and algorithms and derived time series of weekly coordinates of these stations. The station coordinate repeatabilities of daily solutions with respect to the weekly ones reach $1 \mathrm{~mm}$ in the north and east components and $3 \mathrm{~mm}$ in the up component. The standard deviations of the north and east coordinate residual weighted average of our solution with respect to those of the IG1 combined solution of the first IGS data reprocessing campaign are about $1-2 \mathrm{~mm}$ and those of the up component are about 3-4 $\mathrm{mm}$ for the whole time span. Vertical velocities of GPS stations having a tracking history longer than $2.5 \mathrm{yr}$ were computed and compared with the estimates from tide gauge data and some other GPS-derived solutions. Some examples of different types of station vertical velocities such as prominent secular height trend, trend changes caused by various geophysical processes and zero height trend are presented and discussed. The comparison of the vertical velocities of GPS stations of our solution with those of some other solutions including the recent (ULR3 


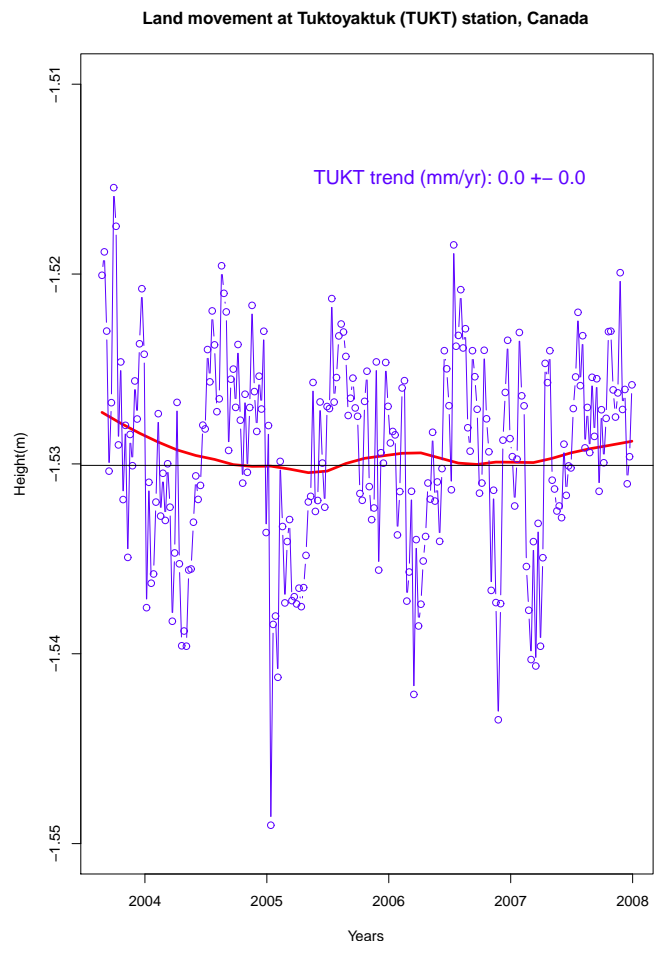

Fig. 16. The Canadian GPS station Tuktoyaktuk has close to zero height trend over the years 2003.7-2008.0.

and ULR5) solutions of Université de La Rochelle indicates that the accuracy of the vertical velocities is below $1 \mathrm{~mm} \mathrm{yr}^{-1}$ for the most GPS stations of our solution. The height time series for the GPS stations co-located at tide gauges and vertical velocities at these stations can be used to correct the estimates of regional and global sea level changes based on tide gauge data. It is planned to perform a new reprocessing of continuous GPS data at longer time span (1994-2011) for an increased number of GPS stations at tide gauges by using newer models and ITRF2008 as a priori terrestrial reference frame within the second IGS data reprocessing campaign. This will allow computation of a longer time series of station coordinates for a larger number of GPS stations with an increased accuracy of vertical velocity.

Acknowledgements. GPS data available from IGS, TIGA and some other stations and agencies were used in this study. The research was supported by German Ministry of Education and Research (BMBF) within the GEOTECHNOLOGIEN geoscientific $R \& D$ programme (SEAVAR Project). The study described in Sect. 4 was performed by N. Schön. The authors are grateful to T. Schöne (GFZ) for useful discussions, K. Snopek (GFZ) for some advices related to LaTeX use, the reviewers (J.R. Ray, A. SantamaríaGómez and anonymous one) and topical editor C. Gaina for their valuable comments and suggestions that improved the paper.

Edited by: C. Gaina

\section{References}

Altamimi, Z., Collilieux, X., Legrand, J., Garayt, B., and Boucher, C.: ITRF2005: A new release of the International Terrestrial Reference Frame based on time series of station positions and Earth Orientation Parameters, J. Geophys. Res., 112, B09401, doi:10.1029/2007JB004949, 2007.

Altamimi, Z., Collilieux, X., and Métivier, L.: ITRF2008: an improved solution of the international terrestrial reference frame, J. Geodesy, 85, 457-473, doi:10.1007/s00190-011-0444-4, 2011.

Altamimi, Z., Sillard, P., and Boucher, C.: ITRF2000: A new release of the International Terrestrial Reference Frame for earth science applications, J. Geophys. Res., 107(B10), 2214, doi:10.1029/2001JB000561, 2002.

Bar-Sever, Y. E.: A new model for GPS yaw attitude, J. Geodesy, 70, 714-723, doi:10.1007/BF00867149, 1996.

Beutler, G., Brockmann, E., Hugentobler, U., Mervart, L., Rothacher, M., and Weber, R.: Combining consecutive short arcs into long arcs for precise and efficient GPS orbit determination, J. Geodesy, 70, 287-299, doi:10.1007/BF00867349, 1996.

Blewitt, G. and Lavallée, D.: Effect of annual signals on geodetic velocity, J. Geophys. Res., 107, B7, 2145, doi:10.1029/2001JB000570, 2002.

Boehm, J., Niell, A. E., Tregoning, P., and Schuh, H.: Global Mapping Functions (GMF): a new empirical mapping function based on numerical weather model data, Geophys. Res. Lett., 33, L07304, doi:10.1029/2005GL025546, 2006.

Boehm, J., Heinkelmann, R., and Schuh, H.: Short Note: a global model of pressure and temperature for geodetic applications, J. Geodesy, 81, 679-683, doi:10.1007/s00190-007-0135-3, 2007.

Bouin, M. N. and Wöppelmann, G.: Land motion estimates from GPS at tide gauges: a geophysical evaluation, Geophys. J. Int., 180, 193-209, doi:10.1111/j.1365-246X.2009.04411.x, 2010.

Buble, G., Bennett, R. A., and Hreinsdóttir, S.: Tide gauge and GPS measurements of crustal motion and sea level rise along the eastern margin of Adria, J. Geophys. Res., 115, B02404, doi:10.1029/2008JB006155, 2010.

Cleveland, R. B., Cleveland, W. S., McRae, J. E., and Terpenning, I.: STL: A seasonal-trend decomposition procedure based on Loess, Journal of Official Statistics, 6, 3-73, 1990.

Collilieux, X. and Wöppelmann, G.: Global sea-level rise and its relation to the terrestrial reference frame, J. Geodesy, 85, 9-22, doi:10.1007/s00190-010-0412-4, 2011.

Collilieux, X., Métivier, L., Altamimi, Z., van Dam, T., and Ray, J.: Quality assessment of GPS reprocessed terrestrial reference frame, GPS Solut., 15, 219-231, doi:10.1007/s10291-010-01846, 2011.

Déry, S. J., Mlynowski, T. J., Hernández-Henríquez, M. A., and Straneo, F.: Interannual variability and interdecadal trends in Hudson Bay streamflow, J. Marine Syst., 88, 341-351, doi:10.1016/j.jmarsys.2010.12.002, 2011.

Dill, R.: Hydrological model LSDM for operational Earth rotation and gravity field variations, Scientific Technical Report, GFZ Potsdam, STR08/09, 37 pp., doi:10.2312/GFZ.b103-08095, 2008.

Dow, J.M., Neilan, R.E., and Rizos, C.: The international GNSS Service in a changing landscape of Global Navigation Satellite Systems, J. Geodesy 83, 191-198, doi:10.1007/s00190-0080300-3, 2009. 
EM-DAT: The OFDA/CRED International Disaster Database, Université Catholique de Louvain, Belgium, Brussels, available at: http://www.emdat.be, 2011.

Emery, K. O. and Aubrey, D. G.: Sea levels, land levels, and tide gauges, Springer, New York, 1991.

Esquivel, R., Hernández, A., and Zermeño, M.E.: GPS for Subsidence Detection, the Case Study of Aguascalientes, in: Geodetic Deformation Monitoring: from Geophysical to Engineering Roles, International Association of Geodesy Symposia, Springer, 131, 254-258, doi:10.1007/978-3-540-38596-7_31, 2006.

$\mathrm{Fu}$, Y., Freymueller, J. T., and van Dam, T.: The effect of using inconsistent ocean tidal loading models on GPS coordinate solutions, J. Geodesy, 86, 409-421, doi:10.1007/s00190-011-0528-1, 2012.

Ge, M., Gendt, G., Dick, G., and Zhang, F. P.: Improving carrierphase ambiguity resolution in global GPS network solutions, J. Geodesy, 79, 103-110, doi:10.1007/s00190-005-0447-0, 2005.

Ge, M., Gendt, G., Dick, G., Zhang, F. P., and Rothacher, M.: A new data processing strategy for huge GNSS global networks, J. Geodesy, 80, 199-203. doi:10.1007/s00190-006-0044-x, 2006.

Gendt, G., Dick, G., Mai, W., Nischan, T., and Sommerfeld, W.: Nutzerhandbuch zum Programmsystem EPOS. P.V2 (Earth Parameters and Orbit determination System) für die Analyse von GPS-Daten, GeoForschungsZentrum Potsdam, Bereich "Rezente Kinematik und Dynamik der Erde", 18 February 1994.

Goebell, S. and King, M. A.: Effects of azimuthal multipath asymmetry on long GPS coordinate time series, GPS Solut., 15, 287297, doi:10.1007/s10291-011-0227-7, 2011.

Isoda, Y., Kitamura, F., and Murakami, T.: Interannual variations of the yearly mean sea level around the Japanese islands, Bulletin of Fisheries Sciences, Hokkaido University, 55, 85-95, ISSN:13461842, 2004.

Kaniuth, K., Häfele, P., and Sánchez, L.: Subsidence of the permanent GPS station Bogotá, in: Vertical Reference Systems. IAG Symposia, edited by: Drewes, H., Dodson, A., Fortes, L. P., Sánchez, L., and Sandoval, P., Springer, 124, 56-59, 2002.

King, M. A., Bevis, M., Wilson, T., Johns, B., and Blume, F.: Monument-antenna effects on GPS coordinate time series with application to vertical rates in Antarctica, J. Geodesy, 86, 53-63, doi:10.1007/s00190-011-0491-x, 2012.

King, M. A. and Watson, C. S.: Long GPS coordinate time series: multipath and geometry effects, J. Geophys. Res., 115, B04403, doi:10.1029/2009JB006543, 2010.

Kurtenbach, E., Mayer-Gürr, T., and Eicker, A.: Deriving daily snapshots of the Earth's gravity field from GRACE L1B data using Kalman filtering, Geophys. Res. Lett., 36, L17102, doi:10.1029/2009GL039564, 2009.

Larsen, C. F., Motyka, R. J., Freymueller, J. T., Echelmeyer, K. A., and Ivins, E. R.: Rapid uplift of southern Alaska caused by recent ice loss, Geophys. J. Int., 158, 1118-1133, doi:10.1111/j.1365246X.2004.02356.x, 2004.

McCarthy, D. D. and Petit, G. (eds.): IERS Conventions (2003), IERS Technical Note 32, 127 pp., ISBN 3-89888-884-3, 2004.

Munekane, H. and Boehm, J.: Numerical simulation of troposphereinduced errors in GPS-derived geodetic time series over Japan, J. Geodesy, 84, 405-417, doi:10.1007/s00190-010-0376-4, 2010.

Sánchez, L. and Bosch, W.: The role of the TIGA project in the unification of classical height systems, in: Geodetic Reference Systems, edited by: Drewes H., IAG Symposia, vol. 134, Springer,
285-290, doi:10.1007/978-3-642-00860-3_44, 2009.

Santamaría-Gómez, A., Gravelle, M., Collilieux, X., Guichard, M., Martín Míguez, B., Tiphaneau, P., and Wöppelmann, G.: Mitigating the effects of vertical land motion in tide gauge records using a state-of-the-art GPS velocity field, Global Planet, 98-99, 6-17, doi:10.1016/j.gloplacha.2012.07.007, 2012.

Sato, T., Mochizuki, K., Kasahara, J., Fujie, G., Nishisaka, H., and Koresawa, S.: Depth variation of the crustal structure of the subducting plate along the Nankai Trough, off Kii Channel, Japan, Geophys. Res. Lett., 25, 21, 4011-4014, doi:10.1029/1998GL900104, 1998.

Schmid, R., Steigenberger, P., Gendt, G., Ge, M., and Rothacher, M.: Generation of a consistent absolute phasecenter correction model for GPS receiver and satellite antennas, J. Geodesy, 81, 781-798, doi:10.1007/s00190-007-0148-y, available at: ftp://igscb.jpl.nasa.gov/pub/station/general/igs05.atx, last access: 15 January 2009, 2007.

Schöne, T., Schön, N., and Thaller, D.: IGS tide gauge benchmark monitoring pilot project (TIGA): scientific benefits, J. Geodesy, 83, 249-261, doi:10.1007/s00190-008-0269-y, 2009.

Utsu, T.: Catalog of Damaging Earthquakes in the World (Through 1989), Utsu, Tokuji, Tokyo, 243 pp., 1990.

van Dam, T., Wahr, J., Milly, P. C. D., Shmakin, A. B., Blewitt, G., Lavallée, D., and Larson, K. M.: Crustal displacements due to continental water loading, Geophys. Res. Lett., 28, 651-654, doi:10.1029/2000GL012120, 2001.

van Dam, T. M.: NCEP derived 6-hourly, global surface displacements at $2.5 \times 2.5$ degree spacing, updated October 2010 , available at: http://geophy.uni.lu/ncep-loading.html, last access: 23 February 2012.

van Dam, T. M., Blewitt, G., and Heflin, M. B.: Atmospheric pressure loading effects on Global Positioning System coordinate determinations, J. Geophys. Res., 99, 23939-23950, doi:10.1029/94JB02122, 1994.

Verbesselt, J., Hyndman, R., Newnham, G., and Culvenor, D.: Detecting trend and seasonal changes in satellite image time series, Remote Sens. Environ., 114, 106-115, doi:10.1016/j.rse.2009.08.014, 2010.

Verdonck, D.: Contemporary vertical crustal deformation in Cascadia, Tectonophysics, 417, 221-230, doi:10.1016/j.tecto.2006.01.006, 2006.

Williams, S. D. P. and Penna, N. T.: Non-tidal ocean loading effects on geodetic GPS heights, Geophys. Res. Lett., 38, L09314, doi:10.1029/2011GL046940, 2011.

Wöppelmann, G., Letetrel, C., Santamaria, A., Bouin, M.-N., Collilieux, X., Altamimi, Z., Williams, S. D. P., and Martin Miguez, B.: Rates of sea-level change over the past century in a geocentric reference frame Geophys. Res. Lett., 36, L12607, doi:10.1029/2009GL038720, 2009.

Wöppelmann, G., Martin Miguez, B., Bouin, M.-N., and Altamimi, Z.: Geocentric sea-level trend estimates from GPS analyses at relevant tide gauges world-wide, Global Planet. Change, 57, 396-406, doi:10.1016/j.gloplacha.2007.02.002, 2007.

Woodworth, P. L. and Player, R.: The Permanent Service for Mean Sea Level: an update to the 21st century, J. Coastal Res., 19, 287295, 2003.

Wu, J. T., Wu, S. C., Hajj, G. A., Bertiger, W. I., and Lichten, S. M.: Effects of antenna orientation on GPS phase, Manuscr. Geodaet, 18, 91-98, 1993. 
Zhang, F. P., Gendt, G., and Ge, M.: GPS Data Processing at GFZ for Monitoring the Vertical Motion of Global Tide Gauge Benchmarks, GeoForschungsZentrum Potsdam Scientific Technical Report STR07/02, 28 pp., doi:10.2312/GFZ.b103-07025, 2007.
Zhang, F. P., Wünsch, J., van Dam, T., Gendt, G., Ge, M., and Schöne, T.: Vertical Crustal Motion at Tide Gauges Derived by Analyzing GPS Time Series, GeoForschungsZentrum Potsdam Scientific Technical Report STR08/03, 106 pp., doi:10.2312/GFZ.b103-08037, 2008. 